\title{
On tours that contain all edges of a hypergraph
}

\author{
Zbigniew Lonc and Paweł Naroski \\ Faculty of Mathematics and Information Science \\ Warsaw University of Technology \\ Pl. Politechniki 1 \\ 00-661 Warszawa, Poland \\ zblonc@mini.pw.edu.pl, p.naroski@mini.pw.edu.pl
}

Submitted: May 25, 2010; Accepted: Sep 16, 2010; Published: Oct 29, 2010

Mathematics Subject Classification: 05C65, 05C45

\begin{abstract}
Let $H$ be a $k$-uniform hypergraph, $k \geqslant 2$. By an Euler tour in $H$ we mean an alternating sequence $v_{0}, e_{1}, v_{1}, e_{2}, v_{2}, \ldots, v_{m-1}, e_{m}, v_{m}=v_{0}$ of vertices and edges in $H$ such that each edge of $H$ appears in this sequence exactly once and $v_{i-1}, v_{i} \in$ $e_{i}, v_{i-1} \neq v_{i}$, for every $i=1,2, \ldots, m$. This is an obvious generalization of the graph theoretic concept of an Euler tour. A straightforward necessary condition for existence of an Euler tour in a $k$-uniform hypergraph is $\left|V_{\text {odd }}(H)\right| \leqslant(k-2)|E(H)|$, where $V_{\text {odd }}(H)$ is the set of vertices of odd degrees in $H$ and $E(H)$ is the set of edges in $H$. In this paper we show that this condition is also sufficient for hypergraphs of a broad class of $k$-uniform hypergraphs, that we call strongly connected hypergraphs. This result reduces to the Euler theorem on existence of Euler tours, when $k=2$, i.e. for graphs, and is quite simple to prove for $k>3$. Therefore, we concentrate on the most interesting case of $k=3$. In this case we further consider the problem of existence of an Euler tour in a certain class of 3-uniform hypergraphs containing the class of strongly connected hypergraphs as a proper subclass. For hypergraphs in this class we give a sufficient condition for existence of an Euler tour and prove intractability (NP-completeness) of the problem in this class in general.
\end{abstract}

Keywords: uniform hypergraphs, Euler tours, Euler walks

\section{Introduction}

In this paper we study some hypergraph generalization of the graph-theoretic concept of an Euler tour. This concept has been extensively studied and is quite well-understood in the case of graphs. Clearly, there are a few possible extensions of the definition of an Euler tour for hypergraphs. The way we do it is motivated by some past research that we shortly describe beneath. 
By a hypergraph we mean a pair $H=(V, E)$, where $V$ is a finite set and $E$ is a family of some subsets of $V$. The elements of the set $V$ are called vertices of $H$ and the elements of $E$ - its edges. We shall call a hypergraph $k$-uniform if all its edges have exactly $k$ elements. To avoid trivial cases, we assume in this paper that $k \geqslant 2$. Clearly, 2-uniform hypergraphs are just simple graphs.

By a walk (or a $v_{0} v_{\ell}$-walk) of length $\ell$ in a hypergraph $H$ we mean an alternating sequence $\left(v_{0}, e_{1}, v_{1}, e_{2}, v_{2}, \ldots, v_{\ell-1}, e_{\ell}, v_{\ell}\right)$ of vertices and edges of this hypergraph satisfying the following conditions:

(i) the edges $e_{1}, e_{2}, \ldots, e_{\ell}$ are pairwise different,

(ii) $v_{i-1}, v_{i} \in e_{i}$, for $i=1, \ldots, \ell$, and

(iii) $v_{i-1} \neq v_{i}$, for $i=1, \ldots, \ell$.

A walk is called a tour if

(iv) $v_{0}=v_{\ell}$.

If, for some tour (or walk), $e_{1}, e_{2}, \ldots, e_{\ell}$ are all the edges of a hypergraph $H$, then we call such tour (or walk) an Euler tour (or walk) in $H$. Let us observe that if $H$ is a 2-uniform hypergraph (i.e. a graph), then the notions of an Euler walk and tour coincide with their traditional meanings in graph theory. Although Euler tours and walks are investigated in the case of multigraphs too, in the present paper we assume that our hypergraphs do not have multiple edges.

It has to be pointed out that the graph-theoretic concepts of a cycle and a tour can be extended to the case of hypergraphs in many possible ways. The tours we consider in this paper are closely related to Berge-cycles (see Berge [6], p. 155). More precisely, a tour defined in this paper in which all vertices $v_{1}, v_{2}, \ldots, v_{\ell}$ are pairwise different is a Berge-cycle.

The concepts of Euler tours and walks defined this way and some related topics have been studied (however in a different setting) by Bartholdi and Goldsman in a series of papers [2], [3], [4], and [5]. Their research, motivated by some applications in geographic information systems, has been concentrated on so-called triangulated irregular networks. Following terminology we use in this paper, a triangulated irregular network (or a TIN) is a 3-uniform hypergraph whose edge set is the family of vertex sets of faces of some planar graph in which all faces, except possibly the outerface, are triangles. The vertex set of a TIN is just the union of all its edges. If we assume additionally that the TIN has some property, that we call strong connectivity (the definition of this notion will be given later), then the main result proved by Bartholdi and Goldsman (see [3], Corollary 3) says that such TIN has an Euler tour, unless it belongs to a very special family of exceptions (described in their paper). Moreover, they show that all strongly connected TINs have an Euler walk. 
The same result on existence of an Euler walk in strongly connected TINs have been independently proved by Wilson [14] who considered this problem in connection with some issues appearing in integer programming.

The problems of existence and construction of Euler tours and walks in TINs have been also studied in computer graphics where the TINs play an important role in optimizing the processes of transmission and rendering of triangulation data representing large threedimensional graphics (see Arkin et al. [1] and Bartholdi and Goldsman [3]).

It is worth mentioning that a closely related problem of existence of a Hamilton cycle in a hypergraph has been intensively studied recently, especially in the context of extension of Dirac's theorem to hypergraphs (e.g. Katona and Kierstead [9], Rödl, Ruciński and Szemerédi [12] and [13], Kühn and Osthus [11], Keevash et al. [10], Gyárfás et al. [8]). However, most of the results proved in these papers concern cycles (called tight or loose) whose definitions are a bit different from the definition of a Berge-cycle (which is a special kind of a tour defined in this paper).

It is natural to ask a question for which $k$-uniform hypergraphs an Euler tour or walk exists. Unlike in the case of graphs, there is little hope to give a full characterization of $k$-uniform hypergraphs, where $k>2$, that have an Euler tour. Our first theorem supports this claim.

Theorem 1 Let $k>2$. The problem of determining if a given $k$-uniform hypergraph has an Euler tour is NP-complete.

Nevertheless, we are able to give a full characterization of $k$-uniform hypergraphs that have an Euler tour, when they belong to a certain broad class of hypergraphs, that we call strongly connected. A $k$-uniform hypergraph $H$ is strongly connected if it does not have isolated vertices and the graph $G(H)$, whose vertex set is the set $E(H)$ of edges of $H$ and the edge set consists of pairs $\{e, f\} \subseteq E(H)$ such that $|e \cap f|=k-1$, is connected. We call $G(H)$ the strong connectivity graph of the hypergraph $H$. When $k=2$, the notion of strong connectivity coincides with the ordinary graph connectivity. By the degree of a vertex $v$ in a hypergraph $H$ we mean the number $d_{H}(v)$ of the edges of $H$ containing $v$, i.e. $d_{H}(v)=|\{e \in E(H): v \in e\}|$. We sometimes write $d(v)$ instead of $d_{H}(v)$ whenever it does not lead to a confusion. The set of vertices of $H$ of odd (respectively even) degrees is denoted by $V_{\text {even }}(H)$ (resp. $\left.V_{\text {odd }}(H)\right)$.

In the case of strongly connected hypergraphs we prove that a certain simple to prove necessary condition for existence of an Euler tour is also sufficient. Here is our main result included in Section 3.

Theorem 2 Let $k \geqslant 2$. A strongly connected $k$-uniform hypergraph $H$ has an Euler tour if and only if

$$
\left|V_{\text {odd }}(H)\right| \leqslant(k-2)|E(H)| \text {. }
$$

This result reduces to the Euler theorem on existence of Euler tours in connected graphs, when $k=2$, and is quite simple to prove for $k>3$. Therefore, we treat the cases of $k=3$ and $k>3$ separately. In the latter case strong connectivity implies the condition (1) unless $H$ has exactly one edge, so Theorem 2 reduces to the following theorem. 
Theorem 3 Let $k>3$. A strongly connected $k$-uniform hypergraph $H$ has an Euler tour if and only if $|E(H)| \neq 1$.

In the most interesting case of $k=3$ we show slightly more than Theorem 2, we additionally describe the strongly connected hypergraphs that satisfy the condition (1). To formulate this result, we need to introduce a class of 3 -uniform hypergraphs $\mathcal{H}$ which is some kind of a hypergraph analogy of the class of trees in graph theory.

Let $V(H)$ be the vertex set of a hypergraph $H$.

We define $\mathcal{H}$ to be the smallest class of 3 -uniform hypergraphs such that

(i) the one-edge 3-uniform hypergraph belongs to $\mathcal{H}$ and

(ii) if $H \in \mathcal{H}$ and $f$ is a 3 -element set such that for some $x \in f, x \notin V(H)$ and $f-\{x\}$ is a subset of some edge in $H$, then the hypergraph $H+f=(V(H) \cup\{x\}, E(H) \cup\{f\})$ belongs to $\mathcal{H}$.

Clearly, the class of trees consists of all connected graphs with the least possible number of edges, for a fixed number of vertices. Analogously, as we prove in Lemmas 2 and 3 , the family $\mathcal{H}$ consists of all strongly connected 3-uniform hypergraphs $H$ with the least possible number of edges (equal to $|V(H)|-2$ ), for a fixed number of vertices. Here is our theorem characterizing strongly connected 3-uniform hypergraphs with Euler tours proved in Section 3.

Theorem 4 Let $H$ be a strongly connected 3-uniform hypergraph. The following statements are equivalent

(i) H has an Euler tour,

(ii) $\left|V_{\text {odd }}(H)\right| \leqslant|E(H)|$,

(iii) $H \notin \mathcal{H}$ or $H$ has a vertex with even degree.

We conclude Section 3 with a result solving the problem of existence of an Euler walk in strongly connected hypergraphs.

Theorem 5 Let $k \geqslant 3$. Every strongly connected $k$-uniform hypergraph has an Euler walk.

Theorems 4 and 5 contain as a special cases the results of Bartholdi and Goldsman [3] and Wilson [14] mentioned above concerning existence of Euler tours and walks in TINs.

Section 4 of this paper is devoted to the problem of existence of an Euler tour in a certain class of 3-uniform hypergraphs containing the class of strongly connected hypergraphs as a proper subclass. To define this class and formulate the main results proved in Section 4, we need to introduce a few notions.

By the skeleton $S(H)$ of a 3-uniform hypergraph $H$ we mean a graph whose edge set is $\{e \cap f: e, f \in E(H)$ and $|e \cap f|=2\}$. The set of vertices of $S(H)$ is the union of its 
edges. A strong component of a 3-uniform hypergraph $H$ is a hypergraph whose edges form a component in the strong connectivity graph $G(H)$. Finally, a wheel $W_{\ell}$, where $\ell \geqslant 3$, is a 3 -uniform hypergraph whose strong connectivity graph is an $\ell$-edge cycle and there is a vertex which belongs to all the edges of $W_{\ell}$.

We prove (see Lemma 8) that 3-uniform strongly connected hypergraphs have connected skeletons. The class of 3-uniform hypergraphs with a connected skeleton is much broader than the class of 3-uniform strongly connected hypergraphs. Here is the main result of Section 4.

Theorem 6 Let $H$ be a 3-uniform hypergraph whose skeleton is connected. If every strong component of $H$ is neither a member of $\mathcal{H}$ having all vertices of odd degree nor a wheel, then $H$ has an Euler tour.

We observe that the converse of Theorem 6 is not true. Wheels are counterexamples to such implication. We conclude this paper with somewhat surprising negative result showing that the problem to characterize 3-uniform hypergraphs with connected skeletons which have an Euler tour is rather hopeless.

Theorem 7 The problem of determining if a given 3-uniform hypergraph with a connected skeleton has an Euler tour is NP-complete.

\section{Auxiliary terminology and notation}

In this section we define terminology and notation that will be useful in the proofs of our results.

By the order (respectively the size) of a hypergraph $H$ we mean the number of vertices (resp. edges) in $H$. If $v$ is a vertex of $H, e$ is its edge, and $v \in e$, then we say that $v$ and $e$ are incident to each other. To simplify notation, we often write $v_{1} v_{2} \ldots v_{k}$ instead of $\left\{v_{1}, v_{2}, \ldots, v_{k}\right\}$ to denote an edge of a $k$-uniform hypergraph. We say that a hypergraph is induced by a set of edges $E$ if its edge set is $E$ and its vertex set is the union of the edges in $E$.

Let $H$ and $G$ be hypergraphs. We say that $G$ is a subhypergraph of $H$ if $V(G) \subseteq$ $V(H)$ and $E(G) \subseteq E(H)$.

Two hypergraphs $H$ and $G$ are isomorphic if there exists a bijection $\varphi: V(H) \rightarrow$ $V(G)$ such that for every subset $e \subseteq V(H), e \in E(H)$ if and only if $\varphi(e) \in E(G)$.

Let $H$ be a hypergraph and $A \subseteq E(H)$. The hypergraph obtained from $H$ by deleting the edges of $A$ and the isolated vertices that may occur is denoted by $H-A$. When $A$ contains one element only, we write $H-e$ instead of $H-\{e\}$ to simplify notation.

For the same reason, in the notation used for walks and tours, we often omit parenthesis and comas. For example, instead of $C=\left(v_{0}, e_{1}, v_{1}, e_{2}, v_{2}, \ldots, v_{\ell-1}, e_{\ell}, v_{\ell}\right)$, we write $C=$ $v_{0} e_{1} v_{1} e_{2} v_{2} \ldots v_{\ell-1} e_{\ell} v_{\ell}$. If $C$ is a tour, then, for any $i=1, \ldots, \ell$, the sequence

$$
v_{i} e_{i+1} v_{i+1} e_{i+2} v_{i+2} \ldots v_{\ell-1} e_{\ell} v_{\ell} e_{1} v_{1} e_{2} v_{2} \ldots v_{i-1} e_{i} v_{i}
$$


is a tour as well, formally different from $C$. Nevertheless, we identify the tours without stating it explicitly. We say that a walk (or a tour) $C$ passes through a vertex $v_{i}$ if $v_{i}$ is a vertex of this walk (or tour). Similarly, we say that a walk (or a tour) $C$ traverses an edge $e_{i}$ using vertices $v_{i-1}$ and $v_{i}$ if the sequence $v_{i-1} e_{i} v_{i}$ is a subsequence of $C$. For a tour $C$, we sometimes write $v_{i} C v_{i}$ instead of $C$ to indicate that $C$ passes through the vertex $v_{i}$. Similarly, for a $v_{0} v_{\ell}$-walk $P=v_{0} e_{1} v_{1} e_{2} v_{2} \ldots v_{\ell-1} e_{\ell} v_{\ell}$, we sometimes use the notation $v_{0} P v_{\ell}$ to indicate its first and last vertex. For any $0 \leqslant i<j \leqslant \ell$, the sequence $v_{i} e_{i+1} v_{i+1} \ldots v_{j-1} e_{j} v_{j}$ is a walk, which we call a subwalk of the walk $P$ or, more precisely, its $v_{i} v_{j}$-subwalk. If $P^{\prime}$ is any $v_{i} v_{j}$-subwalk of $P$, then we sometimes write $P=v_{0} e_{1} v_{1} \ldots v_{i} P^{\prime} v_{j} \ldots v_{\ell-1} e_{\ell} v_{\ell}$.

\section{Euler tours in strongly connected hypergraphs}

Let us start with the proof of the result demonstrating intractability of the general problem of determining existence of an Euler tour in a $k$-uniform hypergraph, when $k>2$.

Proof of Theorem 1. The problem is in the class NP because verification if a given alternating sequence of vertices and edges of a hypergraph is an Euler tour can be done in polynomial time.

We shall now show a reduction from the problem of determining if a given 3-regular graph has a Hamilton cycle, which is NP-complete (see [7]), to our problem.

Let $G$ be a 3 -regular graph. We define a $k$-uniform hypergraph $H$. The vertex set $V(H)=E(G) \cup \bigcup_{v \in V(G)}\left\{w_{v}^{1}, \ldots, w_{v}^{k-3}\right\}$, where the sets $\left\{w_{v}^{1}, \ldots, w_{v}^{k-3}\right\}$ are pairwise disjoint and disjoint from $E(G)$. The edge set $E(H)=\left\{e_{v}: v \in V(G)\right\}$, where $e_{v}$ is the set consisting of the three edges of $G$ incident to $v$ and $k-3$ vertices $w_{v}^{i}$, for $i=1, \ldots, k-3$. Clearly, the construction of $H$ can be done in polynomial time.

Let us assume that $G$ has a Hamilton cycle whose consecutive vertices are $v_{1}, v_{2}, \ldots$, $v_{m}$. Then the sequence $\left(v_{m} v_{1}, e_{v_{1}}, v_{1} v_{2}, e_{v_{2}}, v_{2} v_{3}, \ldots, v_{m-2} v_{m-1}, e_{v_{m-1}}, v_{m-1} v_{m}, e_{v_{m}}, v_{m} v_{1}\right)$ is an Euler tour in $H$.

Conversely, let $\left(u_{0}, e_{v_{1}}, u_{1}, e_{v_{2}}, u_{2}, \ldots, u_{m-1}, e_{v_{m}}, u_{0}\right)$ be an Euler tour in $H$. By the definition of a tour, $u_{i} \in e_{v_{i}} \cap e_{v_{i+1}}$, for $i=1,2, \ldots, m-1$, and $u_{0} \in e_{v_{m}} \cap e_{v_{1}}$. Hence, by the definition of the sets $e_{v}, u_{i}=v_{i} v_{i+1}$, for $i=1,2, \ldots, m$, and $u_{0}=v_{m} v_{1}$ are edges in $G$. Therefore $v_{1}, v_{2}, \ldots, v_{m}$ are consecutive vertices of a Hamilton cycle in $G$.

Let us show now some necessary conditions for existence of Euler tours and Euler walks in $k$-uniform hypergraphs.

Proposition 1 Let $H$ be a $k$-uniform hypergraph, $k \geqslant 2$, and let $a, b \in V(H), a \neq b$.

(i) If $H$ has an Euler tour, then

$$
\sum_{v \in V(H)}\left\lfloor\frac{d(v)}{2}\right\rfloor \geqslant|E(H)| .
$$

(ii) If $H$ has an Euler ab-walk, then 


$$
\left\lfloor\frac{d(a)+1}{2}\right\rfloor+\left\lfloor\frac{d(b)+1}{2}\right\rfloor+\sum_{v \in V(H)-\{a, b\}}\left\lfloor\frac{d(v)}{2}\right\rfloor \geqslant|E(H)|+1 .
$$

(iii) The inequality (2) is equivalent to

$$
\left|V_{\text {odd }}(H)\right| \leqslant(k-2)|E(H)| \text {. }
$$

Proof. Let $C$ be an Euler tour in a $k$-uniform hypergraph $H$. If $v_{i}$ and $v_{j}$ are two occurrences of the same vertex $v \in V(H)$ in $C$, then $e_{i}, e_{i+1}, e_{j}, e_{j+1}$ are pairwise different edges of $H$ incident to $v$. In other words, each occurrence of $v$ uses up two edges incident to it, so $v$ can occur in $C$ at most $\lfloor d(v) / 2\rfloor$ times. On the other hand, the total number of occurrences of vertices in an Euler tour is $|E(H)|$, so the inequality (2) holds.

The proof of the inequality (3) is similar to the proof of (2).

The following argument proves (4)

$$
\begin{aligned}
& \sum_{v \in V(H)}\left\lfloor\frac{d(v)}{2}\right\rfloor=\sum_{v \in V_{\text {odd }}(H)}\left\lfloor\frac{d(v)}{2}\right\rfloor+\sum_{v \in V_{\text {even }}(H)}\left\lfloor\frac{d(v)}{2}\right\rfloor \\
&=\sum_{v \in V_{\text {odd }}(H)} \frac{d(v)-1}{2}+\sum_{v \in V_{\text {even }}(H)} \frac{d(v)}{2}=\frac{1}{2} \sum_{v \in V(H)} d(v)-\frac{1}{2}\left|V_{\text {odd }}(H)\right| \\
&=\frac{k}{2}|E(H)|-\frac{1}{2}\left|V_{\text {odd }}(H)\right| .
\end{aligned}
$$

As it was mentioned in the Introduction (see Theorem 2), the condition (1) turns out to be sufficient for existence of an Euler tour, when we restrict ourselves to the class of strongly connected $k$-uniform hypergraphs. Clearly, we do not need to prove Theorem 2 for $k=2$ because in this case the condition (1) reduces to $V_{\text {odd }}=\emptyset$ and we get the celebrated Euler Theorem characterizing graphs with an Euler tour.

We shall show now that Theorems 3 and 4 imply Theorem 2 .

Proof of Theorem 2. By Proposition 1, we need to show sufficiency only. Obviously, Theorem 2 follows from Theorem 4 when $k=3$. By Theorem 3, to prove Theorem 2 when $k>3$, it suffices to observe that the one-edge $k$-uniform hypergraph does not satisfy the condition (1).

As we have already mentioned, the proof of Theorem 3 is quite straightforward.

Proof of Theorem 3. As one-edge hypergraphs do not have Euler tours, we only need to prove sufficiency. We apply induction on $|E(H)|$.

Let $H$ be a strongly connected $k$-uniform hypergraph containing only the two edges $e$ and $f$. These edges have at least 3 vertices in common. Let $u$ and $v$ be two of them. Then uev $f u$ is an Euler tour in $H$.

Let us assume now that $|E(H)|>2$. It follows easily from the definition of strong connectivity that there is an edge $e \in E(H)$ such that $H^{\prime}=H-e$ is a strongly connected 
hypergraph. Thus, by the induction hypothesis, there exists an Euler tour $C$ in $H^{\prime}$. Let $f$ be an edge in $H^{\prime}$ which has $k-1$ vertices in common with $e$. Let $x$ denote the only vertex of $f$ which does not belong to $e$.

Suppose first that $C$ traverses $f$ using the vertex $x$ and some vertex $u$. Let $P$ be an Euler $x u$-walk in $H^{\prime}-f$ obtained from $C$ by deleting $f$ and let $v$ be any vertex in $e \cap f$ different from $u$. Then $x P$ Pev $f x$ is an Euler tour in $H$. Otherwise, when $C$ traverses $f$ using vertices $u$ and $v$ different from $x$, we define $P$ to be an Euler $u v$-walk in $H^{\prime}-f$ obtained from $C$ by deleting $f$. Let $w$ be any vertex in $e \cap f$ different from $u$ and $v$. Then $u P v e w f u$ is an Euler tour in $H$.

Proving Theorem 4 is much harder. However, this case is, in our opinion, the most interesting one. The rest of this paper is devoted to studying Euler tours in 3-uniform hypergraphs. The following concept will be very useful in studying strongly connected hypergraphs.

An ordering $e_{1}, \ldots, e_{m}$ of the set of edges of a 3-uniform hypergraph $H$ is called a treelike ordering if, for any $i>1$, there is $j<i$ such that $\left|e_{i} \cap e_{j}\right|=2$. Let us denote by $H_{i}$ the hypergraph induced by the edges $e_{1}, \ldots, e_{i}, 1 \leqslant i \leqslant m$. Obviously, $H_{m}=H$.

Clearly, $\left|V\left(H_{1}\right)\right|-\left|E\left(H_{1}\right)\right|=2$. Moreover, the hypergraph $H_{i+1}$ has one edge more and at most one vertex more than $H_{i}$, so

$$
\left|V\left(H_{i+1}\right)\right|-\left|E\left(H_{i+1}\right)\right| \leqslant\left|V\left(H_{i}\right)\right|-\left|E\left(H_{i}\right)\right|,
$$

for every $i=1, \ldots, m-1$.

Lemma 1 A 3-uniform hypergraph $H$ has a tree-like ordering of the set of edges if and only if $H$ is strongly connected.

Proof. $\quad \Rightarrow$ Let $e_{1}, \ldots, e_{m}$ be a tree-like ordering of the set of edges of a 3-uniform hypergraph $H$. We shall prove by induction on $i$ that the hypergraph $H_{i}$ is strongly connected. This is obviously true for $i=1$. Suppose the hypergraph $H_{i}, i<m$, is strongly connected. We shall prove that $H_{i+1}$ is. By the definition of the tree-like ordering, the edge $e_{i+1}$ has 2 vertices in common with some edge $e_{j}, j \leqslant i$. Thus, there is an edge in the graph $G\left(H_{i+1}\right)$ joining the vertices $e_{i+1}$ and $e_{j}$, so the graph $G\left(H_{i+1}\right)$ is connected because, by the induction hypothesis, the graph $G\left(H_{i}\right)$ is connected. Hence, the hypergraph $H_{i+1}$ is strongly connected. Consequently, $H=H_{m}$ is strongly connected.

$(\Leftarrow)$ Let $e_{1}$ be any of the edges of a strongly connected 3-uniform hypergraph $H$. We construct a tree-like ordering of the set of edges of $H$ inductively. Let us assume that we have already chosen the edges $e_{1}, \ldots, e_{i}$, where $i<m$. We choose as $e_{i+1}$ any of the remaining edges which has 2 common vertices with at least one of the edges $e_{1}, \ldots, e_{i}$. Such an edge must exist because otherwise there is a partition of the vertex set of the graph $G(H)$ into disjoint nonempty sets $A$ and $B$, where $A=\left\{e_{1}, \ldots, e_{i}\right\}$ and $B=E(H)-A$, such that there are no edges in $G(H)$ with one end in $A$ and the other one in $B$. Thus, the graph $G(H)$ is disconnected which contradicts the strong connectivity of $H$. Hence $e_{1}, \ldots, e_{m}$ is as tree-like ordering of the set of edges of $H$. 
Lemma 2 Let $H$ be a strongly connected 3-uniform hypergraph. Then $|V(H)| \leqslant|E(H)|+$ 2 .

Proof. Let $m$ be the number of edges of the hypergraph $H$ and let $e_{1}, \ldots, e_{m}$ be a tree-like ordering of the set of edges of $H$ whose existence is guaranteed by Lemma 1 . By the inequalities (5),

$$
|V(H)|-|E(H)|=\left|V\left(H_{m}\right)\right|-\left|E\left(H_{m}\right)\right| \leqslant\left|V\left(H_{1}\right)\right|-\left|E\left(H_{1}\right)\right|=2 .
$$

In several next lemmas and propositions we prove a few properties of the family $\mathcal{H}$ defined in the Introduction.

Lemma 3 Let $H$ be a 3-uniform hypergraph. Then $H$ is strongly connected and satisfies the equality $|V(H)|=|E(H)|+2$ if and only if $H \in \mathcal{H}$.

Proof. We denote by $m$ the number of edges of the hypergraph $H$.

$\Rightarrow$ Let $e_{1}, \ldots, e_{m}$ be a tree-like ordering of the set of edges of $H$ whose existence is guaranteed by Lemma 1 . We shall prove by induction on $i$ that $H_{i} \in \mathcal{H}$, for $i=1,2, \ldots, m$. This is obviously true for $i=1$. Suppose $H_{i} \in \mathcal{H}, i<m$. It follows from the definition of a tree-like ordering that the edge $e_{i+1}$ has 2 vertices in common with some edge $e_{j}$, $j<i+1$. By the definition of $\mathcal{H}$, to prove that $H_{i+1} \in \mathcal{H}$ it suffices to show that the only vertex, say $x$, of $e_{i+1}-e_{j}$ is not a vertex of $H_{i}$. Applying our assumption and the inequalities (5), we get

$$
\begin{aligned}
2=|V(H)| & -|E(H)|=\left|V\left(H_{m}\right)\right|-\left|E\left(H_{m}\right)\right| \leqslant\left|V\left(H_{i+1}\right)\right|-\left|E\left(H_{i+1}\right)\right| \\
& \leqslant\left|V\left(H_{i}\right)\right|-\left|E\left(H_{i}\right)\right| \leqslant\left|V\left(H_{1}\right)\right|-\left|E\left(H_{1}\right)\right|=2,
\end{aligned}
$$

so $\left|V\left(H_{i+1}\right)\right|=\left|V\left(H_{i}\right)\right|+\left|E\left(H_{i+1}\right)\right|-\left|E\left(H_{i}\right)\right|=\left|V\left(H_{i}\right)\right|+1$. Hence, $x \notin V\left(H_{i}\right)$ because $V\left(H_{i+1}\right)=V\left(H_{i}\right) \cup\{x\}$. Consequently, $H_{i+1} \in \mathcal{H}$.

We have shown that $H_{i} \in \mathcal{H}$, for every $i=1,2, \ldots, m$, so in particular $H=H_{m} \in \mathcal{H}$. $(\Leftarrow)$ We apply induction on $m$.

The implication is obviously true for $m=1$. Let us assume that $m>1$. The hypergraph $H \in \mathcal{H}$ originates from some hypergraph $H^{\prime} \in \mathcal{H}$ by adding an edge and a vertex in the way described in the definition of $\mathcal{H}$. By the induction hypothesis, $\left|V\left(H^{\prime}\right)\right|=$ $\left|E\left(H^{\prime}\right)\right|+2$ and $H^{\prime}$ is strongly connected. This implies immediately that $H$ is strongly connected because the strong connectivity graph $G(H)$ originates from the connected graph $G\left(H^{\prime}\right)$ by adding a new vertex and joining it to at least one of the vertices of $G\left(H^{\prime}\right)$. Moreover, $|V(H)|=\left|V\left(H^{\prime}\right)\right|+1=\left|E\left(H^{\prime}\right)\right|+2+1=|E(H)|+2$.

An important property of hypergraphs $H \in \mathcal{H}$ with more than one edge is that they contain a subhypergraph, of one of only three types, which is attached to the rest of the hypergraph $H$ in a very restricted way. We introduce now some basic definitions needed to describe this property. 


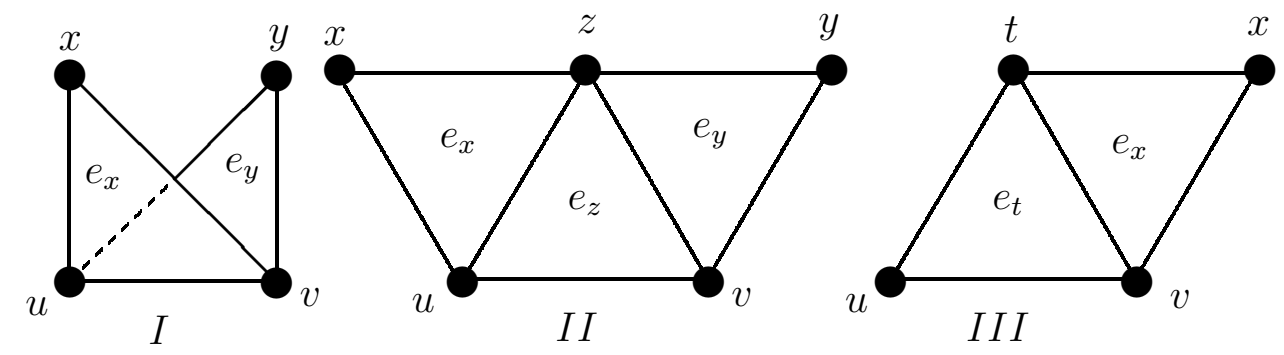

Figure 1: The three types of subhypergraphs.

Let $H$ be a 3-uniform hypergraph. A 2-edge subhypergraph of $H$ induced by some edges $u v x$ and $u v y$ is a subhypergraph of type $\mathbf{I}$ if $d_{H}(x)=d_{H}(y)=1$. A 3-edge subhypergraph of $H$ induced by some edges $u v z, u z x$ and $v z y$ is a subhypergraph of type II if $d_{H}(x)=d_{H}(y)=1$ and $d_{H}(z)=3$. Finally, a 2-edge subhypergraph of $H$ induced by some edges $u v t$ and $v t x$ is a subhypergraph of type III if $d_{H}(x)=1$ and $d_{H}(t)=2$. All three types of subhypergraphs are depicted in Figure 1 . The vertices of these subhypergraphs different from $u$ and $v$ will be called labeled vertices of the subhypergraphs. The vertices $u$ and $v$ will be called connectors of the subhypergraphs. We observe that if $B$ is a subhypergraph of $H$ of type I, II, or III, then each edge in $H$ containing a labeled vertex of $B$ is an edge of $B$. We shall denote by $e_{x}$ the edge in a subhypergraph of type I, II, or III incident with $x$, by $e_{y}$ the edge in a subhypergraph of type I or II incident with $y$, by $e_{t}$ the edge in a subhypergraph of type III incident with $t$ and different from $e_{x}$, and by $e_{z}$ the edge in a subhypergraph of type II incident with $z$ and different from $e_{x}$ and $e_{y}$.

The following proposition is a direct consequence of the definitions of the subhypergraphs of types I, II, and III, the definition of strong connectivity, and Lemma 3.

Proposition 2 Let $H$ be a strongly connected 3-uniform hypergraph of size larger than 3, $B$ a subhypergraph of $H$ of type $I$, II, or III with connectors $u$ and $v$, and $H^{\prime}=H-B$.

(i) There is a vertex $w \in V\left(H^{\prime}\right)$ such that uvw $\in E\left(H^{\prime}\right)$.

(ii) If $H \in \mathcal{H}$ then $H^{\prime} \in \mathcal{H}$.

Here is the lemma describing the property of the hypergraphs in $\mathcal{H}$ mentioned above.

Lemma 4 Every hypergraph $H \in \mathcal{H}$ with more than one edge contains a subhypergraph of type I, II, or III.

Proof. We apply induction on $|E(H)|$.

One can readily verify that the Lemma is true for each of the three (up to isomorphism) hypergraphs in $\mathcal{H}$ with 2 and 3 edges.

Let us assume that $|E(H)|>3$. As $H \in \mathcal{H}$, there exists $H^{\prime} \in \mathcal{H}$ and an edge $e$ in $H$ such that $H=H^{\prime}+e$. By the induction hypothesis, $H^{\prime}$ has a subhypergraph, say $B^{\prime}$, of type I, II, or III. If the labeled vertices of $B^{\prime}$ do not belong to $e$, then $B^{\prime}$ is a 
subhypergraph of $H$ of type I, II, or III. Assume it is not so and denote by $a$ the vertex of $e$ such that $a \notin V\left(H^{\prime}\right)$. We shall consider several cases.

Case 1. $B^{\prime}$ is of the type I.

Let $u v x$ and $u v y$ be the edges of $B^{\prime}$. By symmetry, we can assume that $e=u x a$. Then the edges $u v x$ and $u x a$ induce a subhypergraph of type III in $H$.

Case 2. $B^{\prime}$ is of the type II.

Let $u v z, u z x$, and $v z y$ be the edges of $B^{\prime}$. By symmetry, it suffices to consider the following three subcases.

- If $e=u x a$, then the edges $u z x$ and $u x a$ induce a subhypergraph of type III in $H$.

- If $e=u z a$, then the edges $u z x$ and $u z a$ induce a subhypergraph of type I in $H$.

- If $e=z x a$, then the edges $u z x$ and $z x a$ induce a subhypergraph of type III in $H$.

Case 3. $B^{\prime}$ is of the type III.

Let $u v t$ and $v t x$ be the edges of $B^{\prime}$. There are four subcases to be considered in this case.

- If $e=u t a$, then the edges $u v t, u t a$, and $v t x$ induce a subhypergraph of type II in $H$.

- If $e=v t a$, then the edges $v t x$ and $v t a$ induce a subhypergraph of type I in $H$.

- If $e=t x a$, then the edges $v t x$ and $t x a$ induce a subhypergraph of type III in $H$.

- If $e=v x a$, then the edges $v t x$ and $v x a$ induce a subhypergraph of type III in $H$.

The following simple observation will be used several times later. It is a direct consequence of the definition of strong connectivity of a hypergraph.

Proposition 3 Let $H$ be a strongly connected 3-uniform hypergraph and $|E(H)|>1$. Then there is no edge in $H$ that contains two different vertices of degree 1.

We shall define now a subclass of the class $\mathcal{H}$ consisting of some very special hypergraphs.

Let $\mathcal{R}$ be the smallest class of 3-uniform hypergraphs such that

(i) the 2-edge strongly connected 3-uniform hypergraph belongs to $\mathcal{R}$ and

(ii) if $R \in \mathcal{R}$ and $f$ is a 3 -element set such that, for some $x, y \in f, x \notin V(R), d_{R}(y)=1$, and $f-\{x\}$ is a subset of some edge in $R$, then the hypergraph $R+f$ belongs to $\mathcal{R}$. 
We observe that the way of constructing members of $\mathcal{R}$ described in part (ii) of the definition above is a special case of the construction described in part (ii) of the definition of $\mathcal{H}$. In the definition of $\mathcal{R}$ we additionally require that one of the vertices (named $y$ ) of the added edge $f$ has the degree equal to 1 in the original hypergraph. Since the hypergraph described in part (i) of the definition above is a member of $\mathcal{H}$, the following statement is true.

Proposition 4 The class $\mathcal{R}$ is a subclass of $\mathcal{H}$.

We shall call the members of $\mathcal{R}$ ribbons. Figures 2 and 3 show examples of hypergraphs in $\mathcal{H}$ that are and are not ribbons. (Formally, the edges of these hypergraphs are the triangular faces of the planar graphs depicted in these figures.)
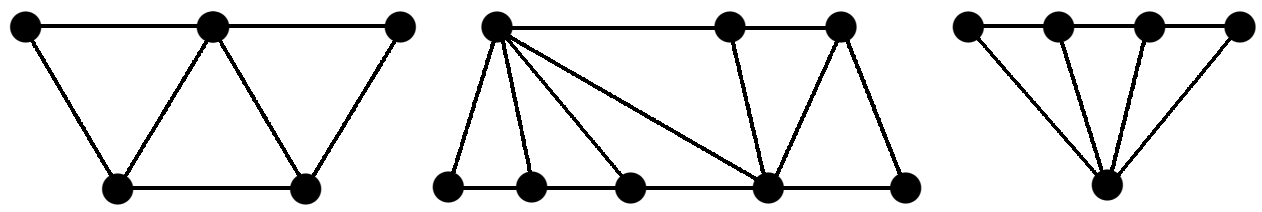

Figure 2: Examples of ribbons.

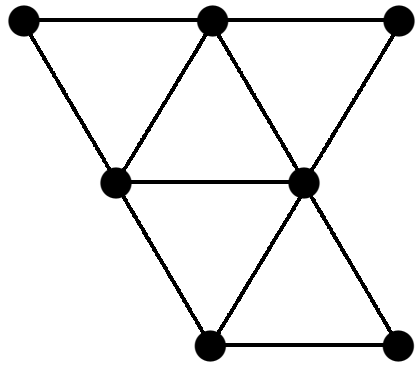

Figure 3: A hypergraph from the class $\mathcal{H}$ which is not a ribbon.

The next two lemmas describe some properties of ribbons.

Lemma 5 Every ribbon has exactly two vertices of degree 1 and at least one vertex with even degree.

Proof. We apply induction on the size of the ribbon.

The unique, up to isomorphism, two-edge ribbon has exactly two vertices of degree 1 and two vertices of degree 2 .

Let us assume now that a ribbon $R$ has more than two edges. By the definition of $\mathcal{R}$, there exist a ribbon $R^{\prime}$ and $f \in E(R)$ such that $R=R^{\prime}+f$, for some $x, y \in f, x \notin V\left(R^{\prime}\right)$, $d_{R^{\prime}}(y)=1$, and $f-\{x\}$ is a subset of some edge, say $e$, in $R^{\prime}$. Obviously, $d_{R}(y)=2$, so $R$ has a vertex with even degree. Moreover, $d_{R}(x)=1$. By the induction hypothesis, $R^{\prime}$ has exactly two vertices of degree 1 . Clearly, $y$ is one of them. Let $a$ be the other one and 
let $z$ be the vertex in $f$ different from $x$ and $y$. We observe that $d_{R^{\prime}}(z)>1$ for otherwise two vertices $\left(y\right.$ and $z$ ) of the edge $e$ have their degrees equal to 1 in $R^{\prime}$, contradicting to Proposition 3. Thus, $a \neq z$ and consequently, $d_{R}(a)=1$. As the degrees of the vertices in $R$ are not smaller than in $R^{\prime}$, the vertices $a$ and $x$ are the only vertices of degree 1 in $R$.

The two vertices of degree 1 in a ribbon will be called the ends of the ribbon.

Let us observe that, unlike in the case of connected graphs, an Euler tour or walk in a 3-uniform strongly connected hypergraph may not pass through every vertex of the hypergraph.

Lemma 6 Let $R$ be a ribbon and let $a$ and $b$ be its ends.

(i) There is no Euler ab-walk in $R$ which passes through every vertex of $R$.

(ii) For every vertex $v \neq a, b$, there is an Euler ab-walk in $R$ which passes through every vertex of $R$ except $v$.

Proof. Let $m$ be the size of $R$. By Proposition 4 and Lemma 3, the order of $R$ is equal to $m+2$.

An Euler walk in $R$ is a sequence of $m+1$ vertices and $m$ edges. Therefore it can not pass through all $m+2$ vertices of $R$, which completes the proof of (i).

Part (ii) of the Lemma will be shown by induction on $m$. The statement is obviously true for the 2-edge ribbon.

Let us assume now that $m>2$. By the definition of $\mathcal{R}$, the ribbon $R$ is of the form $R^{\prime}+f$, where $R^{\prime} \in \mathcal{R}$, and $f$ is a 3-element set such that for some $x, y \in f, x \notin V\left(R^{\prime}\right)$, $d_{R^{\prime}}(y)=1$, and $f-\{x\}$ is a subset of some edge $e$ in $R^{\prime}$. Clearly, $y$ is one of the ends of the ribbon $R^{\prime}$. Let $a$ be the other one. Then, by Lemma 5, $a$ and $x$ are the ends of the ribbon $R$.

Let $v \neq a, x$. If $v \neq y$, then, by the induction hypothesis, there is an Euler $a y$-walk, say $P$, passing through every vertex in $R^{\prime}$ except $v$. The walk $a P y f x$ is an Euler $a x$-walk in $R$ passing through every vertex in $R$ except $v$, which completes the proof in this case.

Finally, we consider the case of $v=y$. Let $z$ be the vertex in $f$ different from $x$ and $y$ and let $e=y z w$ be an edge in $R^{\prime}$ containing $f-\{x\}$. By the induction hypothesis, there is an Euler ay-walk in $R^{\prime}$ passing through every vertex in $R^{\prime}$ except $z$. This walk has to be of the form $a P^{\prime} w e y$. The walk $a P^{\prime} w e z f x$ is an Euler ax-walk in $R$ passing through every vertex in $R$ except $y$.

The next technical lemma concerning Euler walks and tours in hypergraphs of the class $\mathcal{H}$ has a bit tedious proof. We prove here some stronger statements than those which are necessary to show Theorem 4 . We do it because these stronger statements will be used to show Theorem 6 which concerns existence of Euler tours in a more general class of 3-uniform hypergraphs than the class of strongly connected hypergraphs. This is the reason why we define and study what we call good walks and tours.

We say that an Euler tour (or walk) in a hypergraph $H \in \mathcal{H}$ is good if it passes through all vertices of $H$ of degrees greater than 1 . It turns out that hypergraphs in $\mathcal{H}$ 
with some very special exceptions not only have Euler tours and walks but good tours and walks as well.

Lemma 7 Let $H \in \mathcal{H}$.

(i) If $H$ has a vertex with even degree, then $H$ has a good tour.

(ii) If $a, b \in V(H), a \neq b$, are not the only two vertices in $H$ with even degrees and $H$ is not a ribbon with ends $a$ and $b$, then there is a good ab-walk in $H$.

Proof. We shall prove both statements (i) and (ii) by induction on $|E(H)|$.

There are four hypergraphs (up to isomorphism) in $\mathcal{H}$ with at most 3 edges. It can be easily checked that the Lemma is true for each of them.

Let us assume now that $|E(H)|>3$ and the statements (i) and (ii) hold for hypergraphs in $\mathcal{H}$ with size smaller than $|E(H)|$. By Lemma 4, $H$ contains a subhypergraph $B$ of type I, II, or III. Let $u$ and $v$ be the connectors of $B$. It follows from Proposition 2 that the hypergraph $H^{\prime}=H-B \in \mathcal{H}$ and there is a vertex $w \in V\left(H^{\prime}\right)$ such that $e=u v w \in E\left(H^{\prime}\right)$.

(i) If $H$ has a vertex with even degree and $B$ is of type I or II, then $H^{\prime}$ has a vertex with even degree too because the degrees of the connectors in $B$ are in this case equal to 2 . By the induction hypothesis, $H^{\prime}$ has a good tour, say $C$. This tour passes through $u$ or $v$ because it contains the edge $e$. We can assume without loss of generality that $C$ passes through $v$. Then $v e_{x} u e_{y} v C v$ is a good tour in $H$ if $B$ is of the type I and $v e_{y} z e_{x} u e_{z} v C v$ is a good tour in $H$ if $B$ is of the type II.

We assume now that $B$ is of type III. If $H^{\prime}$ has a good $u v$-walk, say $P$, then $u P v e_{x} t e_{t} u$ is a good tour in $H$. Thus, we assume that $H^{\prime}$ does not have a good $u v$-walk. Let $H^{\prime \prime}$ be a hypergraph obtained from $H^{\prime}$ by adding an edge $f=u v s$, where $s$ is a new vertex, i.e. $s \notin V\left(H^{\prime}\right)$. If $H^{\prime \prime}$ has a good tour, then it traverses the edge $f$ using the vertices $u$ and $v$. Consequently, $H^{\prime}$ has a good $u v$-walk, a contradiction. Thus, $H^{\prime \prime}$ does not have a good tour. By the induction hypothesis applied to $H^{\prime \prime}$, all vertices in $H^{\prime \prime}$ have odd degrees. Therefore $H^{\prime}$ has two vertices $u$ and $v$ with even degrees. By the induction hypothesis again, $H^{\prime}$ has a good tour, say $C$. As the degrees of $u$ and $v$ are even (so larger than 1 ), $C$ passes through both $u$ and $v$. Hence, $v C v e_{x} t e_{t} v$ is a good tour in $H$. This completes the inductive proof of (i).

(ii) Suppose that $a, b \in V(H), a \neq b$, are not the only two vertices in $H$ with even degrees and $H$ is not a ribbon with ends $a$ and $b$. We shall prove that $H$ has a good $a b$-walk. We consider two cases.

Case 1. $B$ is of type I or II.

Subcase (i). $a$ and $b$ are not labeled vertices of $B$.

We observe that in this case the parities of all vertices in $H^{\prime}$ are the same as in $H$. Hence, $a$ and $b$ are not the only two vertices in $H^{\prime}$ with even degrees. By the induction hypothesis, there is a good $a b$-walk in $H^{\prime}$ or $H^{\prime}$ is a ribbon with ends $a$ and $b$. In the former case we define $P$ to be this good $a b$-walk in $H^{\prime}$. It passes through at least one of 
the connectors $u$ or $v$, say $v$, because it contains the edge $e$. In the latter case, i.e. when $H^{\prime}$ is a ribbon with ends $a$ and $b$, we observe that at least one of the connectors $u$ or $v$ is not an end of the ribbon $H^{\prime}$ because otherwise, by the fact that $u, v \in e \in E\left(H^{\prime}\right)$ and $\left|E\left(H^{\prime}\right)\right|>1$ (as $H^{\prime}$ is a ribbon), we get a contradiction with Proposition 3 . Let, say $u$, be a vertex in the ribbon $H^{\prime}$ which is not its end. We define in this case $P$ to be an Euler $a b$-walk in $H^{\prime}$ passing through all vertices of $H^{\prime}$ except $u$ whose existence is guaranteed by Lemma 6. In both cases let $P^{\prime}$ be the part of $P$ from $a$ to $v$ and $P^{\prime \prime}$ the rest of the walk $P$ (from $v$ to $b$ ). The walk $a P^{\prime} v e_{x} u e_{y} v P^{\prime \prime} b$ is a good $a b$-walk in $H$ if $B$ is of the type I and the walk $a P^{\prime} v e_{y} z e_{x} u e_{z} v P^{\prime \prime} b$ is a good $a b$-walk in $H$ if $B$ is of the type II.

Subcase (ii). Exactly one of the vertices $a$ or $b$ is a labeled vertex of $B$.

We can assume without loss of generality that $b$ is a labeled vertex of $B$ and $a$ is not. We shall show first that there is a good au-walk or a good av-walk in $H^{\prime}$. If $a \in V\left(H^{\prime}\right)-\{u, v\}$, then it follows by the induction hypothesis (it is also true when $H^{\prime}$ is a ribbon because, by Lemma 5 , at least one of the vertices $a, u$, or $v$ is not an end of the ribbon). If $a \in\{u, v\}$ and a good $u v$-walk in $H^{\prime}$ does not exist, then, by the induction hypothesis, $u$ and $v$ are the only two vertices in $H^{\prime}$ with even degrees or $H^{\prime}$ is a ribbon with ends $u$ and $v$. The latter case leads to a contradiction with Proposition 3 because $u$ and $v$ are vertices of degree 1 in $H^{\prime}$ that belong to the same edge $e \in E\left(H^{\prime}\right)$. In the former case, by part (i) of Lemma $7, H^{\prime}$ has a good tour. This tour must pass through both $u$ and $v$ because these two vertices have even (so larger than 1 ) degrees. In other words the tour is a good $u u$-walk and a good $v v$-walk. We have shown that there is a good $a u$-walk or a good $a v$-walk $P$ in $H^{\prime}$. We can assume without loss of generality that $P$ is a good $a v$-walk in $H^{\prime}$.

One can readily verify that if the hypergraph $B$ is of type $\mathrm{I}$, then it admits good $v x$ and $v y$-walks that pass through $u$. Similarly, if the hypergraph $B$ is of type II, then it admits good $v x^{-}, v y$-, and $v z$-walks that pass through $u$ and $z$. Extending the walk $P$ by one of these walks, we get a good $a b$-walk in $H$, for every labeled vertex $b$ of $B$ and not labeled vertex $a$.

Subcase (iii). Both $a$ and $b$ are labeled vertices of $B$.

Suppose first that $H^{\prime}$ has a good $u v$-walk, say $P$. If $B$ is of type I, then $x e_{x} u P v e_{y} y$ is a good $x y$-walk in $H$. If $B$ is of type II, then $x e_{x} u P v e_{z} z e_{y} y$ is a good $x y$-walk, $x e_{x} z e_{z} u P v e_{y} z$ is a good $x z$-walk, and $z e_{x} u P v e_{z} z e_{y} y$ is a good $z y$-walk in $H$.

Assume now that $H^{\prime}$ does not have a good $u v$-walk. Let $H^{\prime \prime}$ be a hypergraph obtained from $H^{\prime}$ by adding an edge $f=u v s$, where $s$ is a new vertex, i.e. $s \notin V\left(H^{\prime}\right)$. If $H^{\prime \prime}$ has a good tour, then it traverses the edge $f$ using the vertices $u$ and $v$. Consequently, $H^{\prime}$ has a good $u v$-walk, a contradiction. Thus, $H^{\prime \prime}$ does not have a good tour. By part (i) of Lemma 7 , all vertices in $H^{\prime \prime}$ have odd degrees. Consequently, $u$ and $v$ are the only vertices with even (so larger than 1) degree in $H^{\prime}$. By part (i) of Lemma 7 again, $H^{\prime}$ has a good tour, say $C$. By the definition of a good walk, $C$ passes through both $u$ and $v$. If $B$ is of type I, then $x e_{x} u C u e_{y} y$ is a good $x y$-walk in $H$. If $B$ is of type II, then $x e_{x} u C u e_{z} z e_{y} y$ is a good $x y$-walk, $x e_{x} u C u e_{z} v e_{y} z$ is a good $x z$-walk, and $z e_{x} u C u e_{z} v e_{y} y$ is a good $z y$-walk in $H$. 
Case 2. $B$ is of type III.

Subcase (i). $a$ and $b$ are not labeled vertices of $B$.

Suppose first that $H^{\prime}$ has a good $a b$-walk or $H^{\prime}$ is a ribbon with ends $a$ and $b$. We denote by $P$ a good $a b$-walk in $H^{\prime}$ in the former case and an Euler $a b$-walk passing through all vertices of $H^{\prime}$ except $u$ or $v$ in the latter case (such a walk must exist by Lemma 6 because at least one of the vertices $u, v$ is not an end of the ribbon $H^{\prime}$, what follows from Proposition 3). If $P$ passes through both vertices $u$ and $v$, then we denote by $P^{\prime}$ the part of $P$ from $a$ to $v$ and by $P^{\prime \prime}$ the rest of the walk $P$ (from $v$ to $b$ ). Then $a P^{\prime} v e_{t} t e_{x} v P^{\prime \prime} b$ is a good $a b$-walk in $H$. If $P$ does not pass through both vertices $u$ and $v$, then it must traverse the edge $e$ using either $w$ and $u$ or $w$ and $v$. We shall consider the former case only because the latter one is very similar. We can assume without loss of generality that $w$ precedes $e$ and $u$ succeeds $e$ in the $a b$-walk $P$. In other words, $P=a P^{\prime} w e u P^{\prime \prime} b$, where $P^{\prime}$ and $P^{\prime \prime}$ are appropriate parts of the walk $P$. Then $a P^{\prime}$ weve $e_{x} t e_{t} u P^{\prime \prime} b$ is a good $a b$-walk in $H$.

We assume now that there is no good $a b$-walk in $H^{\prime}$ and $H^{\prime}$ is not a ribbon with ends $a$ and $b$. By the induction hypothesis, $a$ and $b$ are the only vertices with even degrees in $H^{\prime}$.

First we assume that $v \notin\{a, b\}$. Then the vertex $v$ has an odd degree in $H^{\prime}$, so it has an even degree in $H-e_{x} \in \mathcal{H}$. Thus, there exists a good $a b$-walk $P$ in $H-e_{x}$, by the induction hypothesis and the fact that $t$ has degree 1 in $H-e_{x}$ (so, by Lemma 5 , $H-e_{x}$ is not a ribbon with ends $a$ and $b$ ). This walk has to traverse the edge $e_{t}$ using the vertices $u$ and $v$, say in this order. Hence, $P=a P^{\prime} u e_{t} v P^{\prime \prime} b$, where $P^{\prime}$ and $P^{\prime \prime}$ are appropriate parts of the walk $P$. Then $a P^{\prime} u e_{t} t e_{x} v P^{\prime \prime} b$ is a good $a b$-walk in $H$.

We assume now that $v \in\{a, b\}$, say $v=a$. By the induction hypothesis, there exists a good $t b$-walk $P$ in $H-e_{x}$ or $H-e_{x}$ is a ribbon with ends $t$ and $b$. In the latter case let $P$ be a $t b$-walk passing through all vertices of $H-e_{x}$ except $v$ (it exists by Lemma 6 , as $v \neq t, b)$. The walk $v e_{x} t P b$ is a good $v b$-walk in $H$.

Subcase (ii). Exactly one of the vertices $a$ or $b$ is a labeled vertex of $B$.

We can assume without loss of generality that $b$ is a labeled vertex of $B$ and $a$ is not.

Let us start with the case of $b=t$ and $a \neq u$. As $a$ and $b$ are not the only vertices with even degrees in $H, a$ and $u$ are not the only vertices with even degrees in $H^{\prime}$. Thus, by the induction hypothesis, there exists a good $a u$-walk in $H^{\prime}$ or $H^{\prime}$ is a ribbon with ends $a$ and $u$. We define $P$ to be a good $a u$-walk in $H^{\prime}$ in the former case and an $a u$-walk passing through every vertex of $H^{\prime}$ except $v$ in the latter case (such a walk exists by Lemma 6 because $v \neq a, u$, by Proposition 3). Then $a P u e_{t} v e_{x} t$ is a good $a t$-walk in $H$.

Let now $b=t$ and $a=u$. As $a$ and $b$ are not the only vertices with even degrees in $H$, some vertex in $H^{\prime}$ has an even degree. Thus, by part (i) of Lemma 7, there exists a good tour $C$ in $H^{\prime}$. It passes through $u$ or $v$ because it contains the edge $e$. Then $u C u e_{t} v e_{x} t$ or $u e_{t} v C v e_{x} t$, respectively, is a good $u t$-walk in $H$.

Finally, let us consider the case of $b=x$. We observe that $H-e_{x}$ is not a ribbon with ends $a$ and $t$ because otherwise, by the definition of a ribbon, $H$ is a ribbon with ends $a$ and $b=x$, contrary to our assumption. Thus, by the induction hypothesis, there exists a good at-walk, say $P$, in $H-e_{x} \in \mathcal{H}$. As $t, v \in e_{t}$, by Proposition 3 , the degree of $v$ in 
$H-e_{x}$ is larger than 1 . Hence the at-walk $P$ passes through $v$, so the walk $a$ Pte $e_{x} x$ is a good $a x$-walk in $H$.

Subcase (iii). Both $a$ and $b$ are labeled vertices of $B$.

We can assume without loss of generality that $a=x$ and $b=t$. As $t, v \in e_{t}$ and the degree of $b=t$ in $H-e_{x} \in \mathcal{H}$ is 1 , by Proposition 3, the degree of $v$ in $H-e_{x}$ is larger than 1. By the induction hypothesis, there exists a good $v t$-walk, say $P$, in $H-e_{x}$. The walk $x e_{x} v P t$ is a good $x t$-walk in $H$.

Lemma 7 allows us to characterize hypergraphs in $\mathcal{H}$ with Euler tours and walks quite completely.

Corollary 1 Let $H \in \mathcal{H}$. The following conditions are equivalent

(i) H has a good tour,

(ii) $H$ has an Euler tour,

(iii) $H$ has a vertex with even degree.

Proof. Since the implication (i) $\Rightarrow$ (ii) is obvious by the definition of a good tour and the implication (iii) $\Rightarrow$ (i) has been shown in Lemma 7 , it remains to prove that (ii) $\Rightarrow$ (iii).

Suppose (ii) holds. By Lemma 3 and Proposition 1,

$$
|E(H)|=|V(H)|-2=\left|V_{\text {odd }}(H)\right|+\left|V_{\text {even }}(H)\right|-2 \leqslant|E(H)|+\left|V_{\text {even }}(H)\right|-2,
$$

SO

$$
\left|V_{\text {even }}(H)\right| \geqslant 2 \text {, }
$$

which proves (iii).

Corollary 2 Let $H \in \mathcal{H}$ and let $a, b \in V(H), a \neq b$. The following conditions are equivalent

(i) there is a good ab-walk in $H$,

(ii) there is an Euler ab-walk in $H$ and $H$ is not a ribbon with ends $a$ and $b$,

(iii) $a$ and $b$ are not the only two vertices in $H$ with even degrees and $H$ is not a ribbon with ends $a$ and $b$.

Proof. The implication (i) $\Rightarrow$ (ii) follows from the definition of a good walk and Lemma 6. The implication (iii) $\Rightarrow$ (i) has been shown in Lemma 7.

To show (ii) $\Rightarrow$ (iii) suppose that $a$ and $b$ are the only two vertices in $H$ with even degrees. By Lemma 3,

$$
\left\lfloor\frac{d(a)+1}{2}\right\rfloor+\left\lfloor\frac{d(b)+1}{2}\right\rfloor+\sum_{v \in V(H)-\{a, b\}}\left\lfloor\frac{d(v)}{2}\right\rfloor
$$

THE ELECTRONiC JouRnal of COMBINATORICS 17 (2010), \#R144 


$$
\begin{gathered}
=\frac{d(a)}{2}+\frac{d(b)}{2}+\sum_{v \in V(H)-\{a, b\}} \frac{d(v)-1}{2} \\
=\frac{3}{2}|E(H)|-\frac{1}{2}|V(H)|+1=|E(H)|<|E(H)|+1,
\end{gathered}
$$

a contradiction with Proposition 1, so $a$ and $b$ are not the only two vertices in $H$ with even degrees.

The next corollary follows directly from the implication (ii) $\Rightarrow$ (iii) in Corollary 2 and Lemma 6.

Corollary 3 Let $H \in \mathcal{H}$ and let $a, b \in V(H), a \neq b$. Then $H$ has an Euler ab-walk if and only if $a$ and $b$ are not the only two vertices in $H$ with even degrees.

Let $H$ be a 3-uniform strongly connected hypergraph of size $m$. By Lemma 1 , there exists a tree-like ordering $e_{1}, \ldots, e_{m}$ of the set of edges of $H$, i.e. such an ordering that, for each $i=2, \ldots, m$, there is a $j<i$ such that $\left|e_{i} \cap e_{j}\right|=2$. For such an ordering of the set of edges of $H$, we construct a hypergraph $T(H) \in \mathcal{H}$. The edges of this hypergraph are $\bar{e}_{1}, \ldots, \bar{e}_{m}$. The vertices of the hypergraph $T(H)$ are denoted by $u[i]$, where $u$ is a vertex of $H$ and $i$ is a nonnegative integer. For every edge $e_{i}=x y z$, the edge $\bar{e}_{i}$ is of the form $x\left[i_{1}\right] y\left[i_{2}\right] z\left[i_{3}\right]$. The values of $i_{1}, i_{2}$ and $i_{3}$ will be specified later. Intuitively, the vertices $u[i]$ in $T(H)$ emerge from the same vertex $u$ in $H$. We define the edges and vertices of $T(H)$ recursively. Let $e_{1}=u v w$. Then $\bar{e}_{1}=u[0] v[0] w[0]$. Suppose we have already defined $\bar{e}_{1}, \ldots, \bar{e}_{i-1}, 2 \leqslant i \leqslant m$. Let $e_{i}=x y z$. We define $j, j<i$, to be the smallest integer such that $\left|e_{i} \cap e_{j}\right|=2$. Let these vertices be $x$ and $y$ and let $e_{j}=x y t$. Then $\bar{e}_{j}=x\left[j_{1}\right] y\left[j_{2}\right] t\left[j_{3}\right]$, where $j_{1}, j_{2}, j_{3}$ are nonnegative integers. We define $\bar{e}_{i}=x\left[j_{1}\right] y\left[j_{2}\right] z[p]$, where $p$ is the smallest nonnegative integer such that $z[p]$ is not a vertex of the edges $\bar{e}_{1}, \ldots, \bar{e}_{i-1}$ yet. We observe that there is a one-to-one correspondence between the edges of $H$ and the edges of $T(H)$. An example of the construction of $T(H)$ is shown in Figure 4 .

Clearly, for a 3-uniform strongly connected hypergraph $H$, the hypergraph $T(H)$ may not be unique. It depends on the choice of a tree-like ordering of the set of edges of $H$.

The next proposition follows directly from the definition of the hypergraph $T(H)$.

Proposition 5 If $H$ is a strongly connected hypergraph, then

(i) $T(H) \in \mathcal{H}$,

(ii) $|E(T(H))|=|E(H)|$,

(iii) $u v w \in E(H)$ if and only if $u\left[i_{1}\right] v\left[i_{2}\right] w\left[i_{3}\right] \in E(T(H))$, for some $i_{1}, i_{2}, i_{3}=0,1, \ldots$,

(iv) if $\bar{P}=\left(v_{0}\left[i_{0}\right], \bar{e}_{1}, v_{1}\left[i_{1}\right], \bar{e}_{2}, v_{2}\left[i_{2}\right], \ldots, v_{\ell-1}\left[i_{\ell-1}\right], \bar{e}_{\ell}, v_{\ell}\left[i_{\ell}\right]\right)$ is a walk in $T(H)$, then $P=$ $\left(v_{0}, e_{1}, v_{1}, e_{2}, v_{2}, \ldots, v_{\ell-1}, e_{\ell}, v_{\ell}\right)$ is a walk in $H$. 

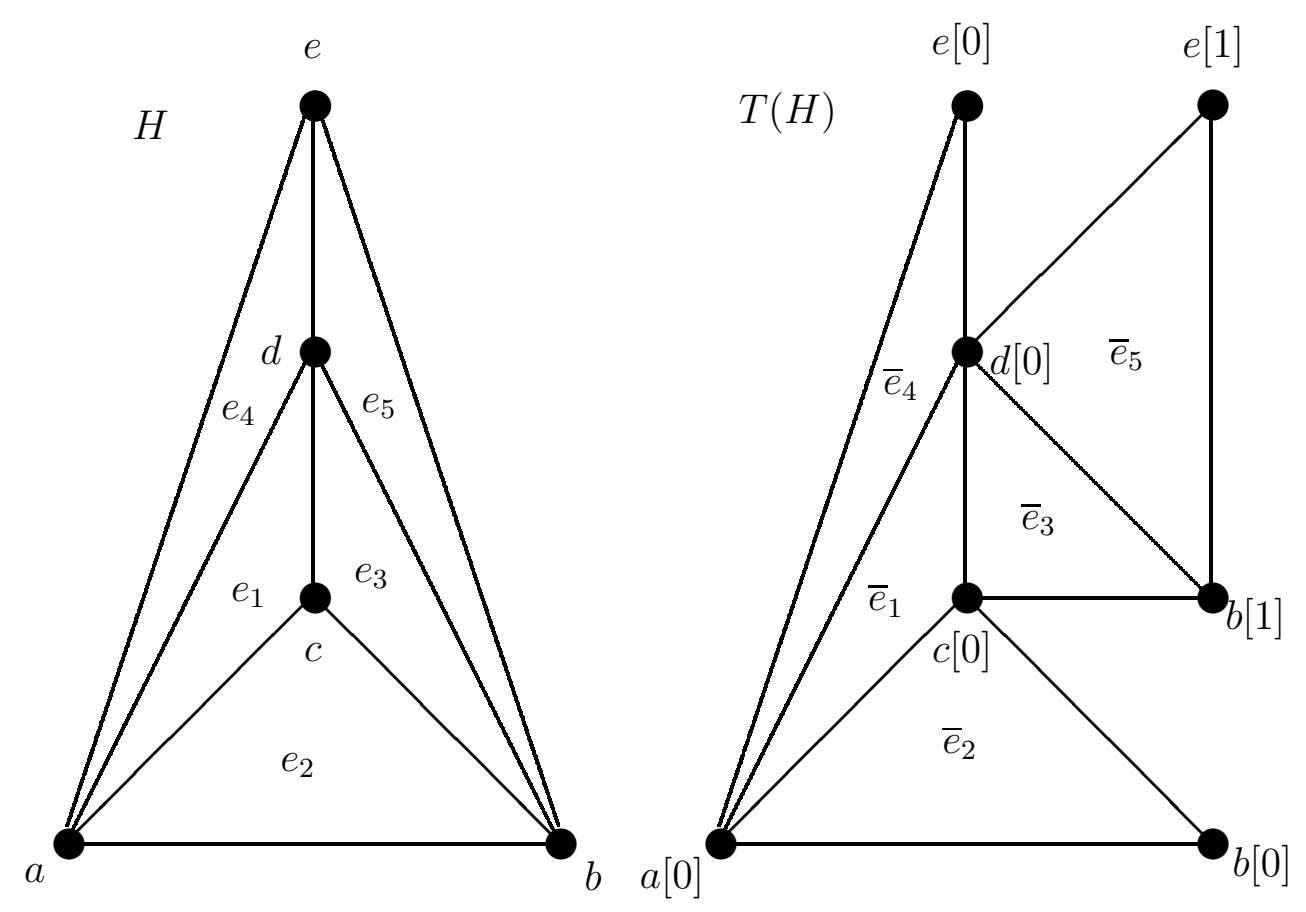

Figure 4: The construction of $T(H)$.

Theorem 4 is now a simple consequence of Corollaries 1, 2, and Proposition 5.

Proof of Theorem 4. By Proposition 1, (i) $\Rightarrow$ (ii).

To show the implication (ii) $\Rightarrow$ (iii), we assume that $H \in \mathcal{H}$ is a hypergraph having no vertex with even degree. Now, by Lemma 3 ,

$$
\left|V_{\text {odd }}(H)\right|=|V(H)|=|E(H)|+2>|E(H)|,
$$

a contradiction with (ii). Thus (ii) $\Rightarrow$ (iii).

To prove the implication (iii) $\Rightarrow$ (i), we assume first that $H \in \mathcal{H}$. By condition (iii), $H$ has a vertex with even degree. We are done in this case by Corollary 1.

Let us assume now that $H \notin \mathcal{H}$. Then, by Lemmas 2 and $3,|V(H)| \leqslant|E(H)|+1$. We consider the hypergraph $T(H)$ defined for some tree-like ordering of the set of edges of $H$. Suppose first $T(H)$ has an Euler tour. By Proposition 5 (iv) and the definition of an Euler tour, the hypergraph $H$ has an Euler tour too. Thus, assume that $T(H)$ does not have an Euler tour. It follows from Proposition 5 (i) and Corollary 1 that all vertices of $T(H)$ have odd degrees. Since, by Proposition 5 (ii) and Lemma 3, $|V(T(H))|=|E(T(H))|+2=$ $|E(H)|+2 \geqslant|V(H)|+1$, there is a vertex $v \in V(H)$ such that $v[0], v[1] \in V(T(H))$. By Corollary 3, $T(H)$ has an Euler $v[0] v[1]$-walk, say $\bar{P}$. By Proposition 5 (iv), $P$ is an Euler tour in $H$.

The results we have already proved allow us to solve the problem of existence of an Euler walk in strongly connected hypergraphs. 

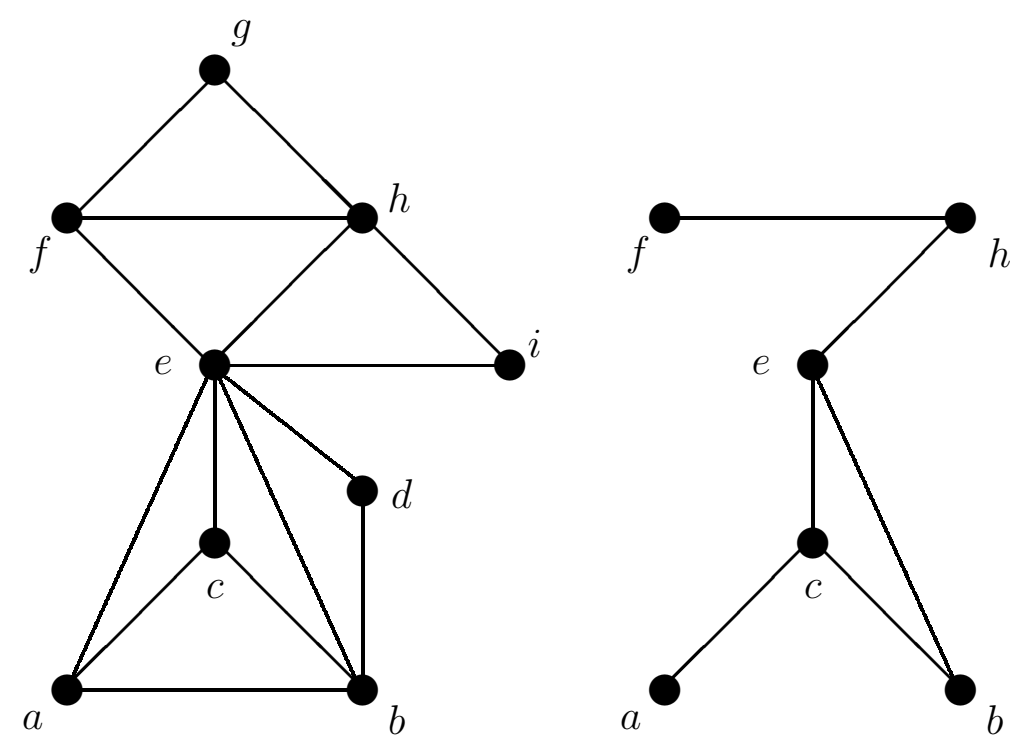

Figure 5: The figure on the left is a 3-uniform hypergraph, say $H$, in which the triangular faces are the edges. The figure on the right is the skeleton $S(H)$ of the hypergraph $H$.

Proof of Theorem 5. As every Euler tour is an Euler walk and the one-edge hypergraph has an Euler walk, the theorem obviously follows from Theorem 3, when $k>3$. For a similar reason, when $k=3$, the theorem is an easy consequence of Theorem 4 and Corollary 3.

\section{Beyond the strong connectivity}

In this section we study existence of Euler tours in the class of 3-uniform hypergraphs with a connected skeleton. The class of strongly connected 3-uniform hypergraphs is a proper subclass of this class.

An example of a 3-uniform hypergraph and its skeleton is shown in Figure 5 . The hypergraph depicted in this figure is an example of a 3-uniform hypergraph which is not strongly connected, but has a connected skeleton.

Lemma 8 The skeleton of a strongly connected 3-uniform hypergraph $H$ is a connected graph.

Proof. We apply induction on the size of $H$.

If $H$ is a one-edge hypergraph, then its skeleton is the graph induced by the empty set of edges, so it is connected. If $H$ has two edges, then its skeleton is a graph induced by one edge which is connected too.

Let us assume now that $H$ has at least three edges. We denote by $e=u v w \in E(H)$ such an edge that $H-e$ is strongly connected. It exists by the definition of a strongly connected hypergraph. Let $f$ be an edge in $H-e$ such that $|f \cap e|=2$. Without loss of 
generality we assume that $f=u v x$, for some vertex $x$ in $H-e$. Since $H-e$ is strongly connected, $f$ is an edge in $H-e$, and $H$ has at least 3 edges, there is an edge, say $g$, in $H-e$ such that $|g \cap f|=2$. Thus, at least one of the vertices $u$ or $v$ belongs to the skeleton of $H-e$, so to the skeleton of $H$ as well. By the induction hypothesis, the skeleton of $H-e$ is connected. The skeleton of $H$ consists of all the edges of the skeleton of $H-e$, the edge $u v$, and, possibly, one or two of the edges $u w, v w$. In each case the skeleton of $H$ is connected because, as we have shown, at least one of the vertices $u$ or $v$ belongs to the skeleton of $H$.

In the most important step (see the proof of Lemma 17) of the reasoning, that leads to the proof of the main result of this section (Theorem 6), we apply induction on $s(H)=$ $|E(H)|-|V(H)|+2$. In the induction step we need, for a 3-uniform strongly connected hypergraph $H$ such that $s(H)>0$, a construction of a strongly connected hypergraph (that we denote by $H_{e}^{z}$ ) such that $s\left(H_{e}^{z}\right)=s(H)-1$ and existence of an Euler tour in $H_{e}^{z}$ implies the existence of an Euler tour in $H$. We describe and study properties of this construction below.

Let $e$ be an edge in a strongly connected 3-uniform hypergraph $H$ and let $e_{1}, e_{2}, \ldots, e_{m}$ be a tree-like ordering of the set of edges of $H$ such that $e=e_{i}$. We call this tree-like ordering $(e, z)$-ordering of the set of edges of $H$ if

(i) $z$ is a vertex of $e$ such that $e-\{z\} \subseteq e_{j}$, for some $j<i$,

(ii) all vertices of $e$ belong to the vertex set of the hypergraph induced by the set of edges $\left\{e_{1}, e_{2}, \ldots, e_{i-1}\right\}$, and

(iii) for every $j>i$, the ordering $e_{1}, e_{2}, \ldots, e_{i-1}, e_{j}, e_{i}, e_{i+1}, \ldots, e_{j-1}, e_{j+1}, \ldots, e_{m}$ is not a tree-like ordering of the set of edges of $H$.

An example of an $(e, z)$-ordering of the set of edges of a hypergraph is shown in Figure 6.

Lemma 9 If $H$ is a 3-uniform strongly connected hypergraph such that

$$
|V(H)|<|E(H)|+2
$$

then $H$ has an $(e, z)$-ordering, for some edge $e \in E(H)$ and vertex $z \in e$.

Proof. Let $e_{1}, e_{2}, \ldots, e_{m}$ be a tree-like ordering of the set of edges of $H$ which exists by virtue of Lemma 1. Suppose that the condition (ii) in the definition of an $(e, z)$ ordering is not satisfied for any edge $e$ for this tree-like ordering. Then, for every edge $e_{i}$, $i=2,3, \ldots, e_{m}$, the edge $e_{i}$ contains a vertex which is in none of the edges $e_{1}, e_{2}, \ldots, e_{i-1}$. Hence, $|V(H)|=\left|e_{1}\right|+m-1=m+2=|E(H)|+2>|V(H)|$, a contradiction. Thus, for every tree-like ordering $e_{1}, e_{2}, \ldots, e_{m}$ of the set of edges of $H$, there is an edge $e=e_{i}$ such that the condition (ii) in the definition of an $(e, z)$-ordering is satisfied.

Let us consider a tree-like ordering $e_{1}, e_{2}, \ldots, e_{m}$ of the set of edges of $H$ such that the index $i$, for which the condition (ii) is satisfied for $e=e_{i}$, is the largest. For this tree-like 


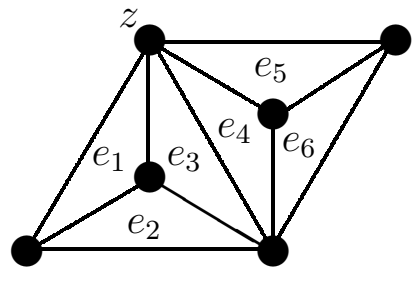

$H$

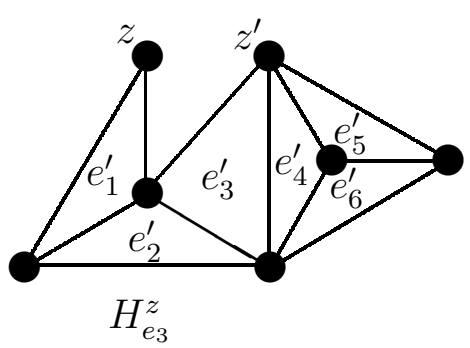

Figure 6: The sequence $e_{1}, e_{2}, e_{3}, e_{4}, e_{5}, e_{6}$ is an $\left(e_{3}, z\right)$-ordering of the set of edges of $H$.

ordering the condition (iii) is satisfied too. By the definition of a tree-like ordering of the set of edges, the edge $e$ has two vertices in common with some edge $e_{j}, j<i$. Let $z$ be the vertex of $e_{i}$ which is none of these two vertices. For such $z$ the condition (i) in the definition of an $(e, z)$-ordering is satisfied. We have shown existence of an $(e, z)$-ordering of the set of edges of $H$.

An $(e, z)$-ordering $e_{1}, e_{2}, \ldots, e_{m}$ is terminal if $e$ is the last edge in this ordering, i.e. $e=e_{m}$. An edge $e \in E(H)$ is substantial if there is an $(e, z)$-ordering of the set of edges of $H$, for some vertex $z \in e$.

The following simple observation will be used several times in the sequel.

Lemma 10 If $e_{1}, e_{2}, \ldots e_{m}$ is an $(e, z)$-ordering of the set of edges of a 3-uniform strongly connected hypergraph $H$, where $e=e_{i}$, then $\left|e_{\ell} \cap e_{j}\right| \neq 2$, for every $\ell<i$ and $j>i$.

Proof. Suppose $\left|e_{\ell} \cap e_{j}\right|=2$, for some $\ell<i$ and $j>i$. Then the ordering

$$
e_{1}, e_{2}, \ldots, e_{i-1}, e_{j}, e_{i}, e_{i+1}, \ldots, e_{j-1}, e_{j+1}, \ldots, e_{m}
$$

is a tree-like ordering of the set of edges of $H$ contradicting the condition (iii) in the definition of an $(e, z)$-ordering of the set of edges of a hypergraph.

Let $e_{1}, e_{2}, \ldots, e_{m}$ be an $\left(e_{i}, z\right)$-ordering of the set of edges of a strongly connected 3-uniform hypergraph $H$ and let $z^{\prime} \notin V(H)$. We define

$$
e_{j}^{\prime}= \begin{cases}e_{j} & \text { if } j<i \text { or } z \notin e_{j} \\ \left(e_{j}-\{z\}\right) \cup\left\{z^{\prime}\right\} & \text { if } j \geqslant i \text { and } z \in e_{j} .\end{cases}
$$

We denote by $H_{e}^{z}$ the hypergraph induced by the edges $e_{1}^{\prime}, e_{2}^{\prime}, \ldots, e_{m}^{\prime}$.

An example of a hypergraph $H_{e}^{z}$ constructed for some $(e, z)$-ordering of the set of edges of a hypergraph $H$ is shown in Figure 6.

In the next three lemmas we assume that the hypergraph $H_{e}^{z}$ is defined for some $(e, z)$-ordering of the set of edges of a strongly connected 3-uniform hypergraph $H$.

Lemma 11 The hypergraph $H_{e}^{z}$ is strongly connected. 
Proof. Let $e_{1}, e_{2}, \ldots, e_{m}$ be an $(e, z)$-ordering of the set of edges of $H$ and let $e=e_{i}$. Hence, by the definition of a tree-like ordering of the set of edges, for every $j>1$, there is $\ell<j$ such that $\left|e_{\ell} \cap e_{j}\right|=2$. By Lemma 1, to show that the hypergraph $H_{e}^{z}$ is strongly connected, it suffices to prove that $e_{1}^{\prime}, e_{2}^{\prime}, \ldots, e_{m}^{\prime}$ is a tree-like ordering of the set of edges of $H_{e}^{z}$.

We shall show first that, for every $1<j \neq i,\left|e_{j}^{\prime} \cap e_{\ell}^{\prime}\right|=2$. This is obviously true for $j<i$. If $z \notin e_{j}$, then $z \notin e_{j} \cap e_{\ell}$, so $e_{j}^{\prime} \cap e_{\ell}^{\prime}=e_{j} \cap e_{\ell}^{\prime}=e_{j} \cap e_{\ell}$ and, consequently, $\left|e_{j}^{\prime} \cap e_{\ell}^{\prime}\right|=2$.

Let us now assume that $j>i$ and $z \in e_{j}$. By Lemma 10, $\ell \geqslant i$. If $z \in e_{\ell}$, then $e_{j}^{\prime} \cap e_{\ell}^{\prime}=\left(\left(e_{j}-\{z\}\right) \cup\left\{z^{\prime}\right\}\right) \cap\left(\left(e_{\ell}-\{z\}\right) \cup\left\{z^{\prime}\right\}\right)=\left(\left(e_{j} \cap e_{\ell}\right)-\{z\}\right) \cup\left\{z^{\prime}\right\}$, so $\left|e_{j}^{\prime} \cap e_{\ell}^{\prime}\right|=2$. If $z \notin e_{\ell}$, then $e_{j}^{\prime} \cap e_{\ell}^{\prime}=\left(\left(e_{j}-\{z\}\right) \cup\left\{z^{\prime}\right\}\right) \cap e_{\ell}=e_{j} \cap e_{\ell}$, so we are done too.

Finally, let $j=i$. By the definition of an $(e, z)$-ordering, $e_{i}-\{z\} \subseteq e_{\ell}$, for some $\ell<i$. Hence $e_{i}^{\prime} \cap e_{\ell}^{\prime}=\left(\left(e_{i}-\{z\}\right) \cup\left\{z^{\prime}\right\}\right) \cap e_{\ell}=e_{i}-\{z\}$, so $\left|e_{i}^{\prime} \cap e_{\ell}^{\prime}\right|=2$.

Lemma 12 Let $P^{\prime}$ be a walk in $H_{e}^{z}$ and let the sequence $P$ be obtained from $P^{\prime}$ by replacing every occurrence of an edge $e_{i}^{\prime} \in E\left(H_{e}^{z}\right)$ by the edge $e_{i} \in E(H)$ and every occurrence of the vertex $z^{\prime}$ by the vertex $z$. Now

(i) $P$ is a walk in $H$,

(ii) if $P^{\prime}$ passes through a vertex $v \neq z^{\prime}$ in $H_{e}^{z}$, then $P$ passes through $v$ in $H$ and if $P^{\prime}$ passes through the vertex $z^{\prime}$ in $H_{e}^{z}$, then $P$ passes through $z$ in $H$.

Proof. We check that the sequence $P$ satisfies the conditions (i)-(iii) in the definition of a walk in a hypergraph. The condition (i) is satisfied because there is a one-to-one correspondence between the edges $e_{i}$ in $H$ and the edges $e_{i}^{\prime}$ in $H_{e}^{z}$. The condition (ii) in the definition of a walk and the condition (ii) of this Lemma hold because, by the definition of $H_{e}^{z}$, if $v \in e_{i}^{\prime}, v \neq z^{\prime}$, then $v \in e_{i}$ and if $z^{\prime} \in e_{i}^{\prime}$, then $z \in e_{i}$. Finally, the condition (iii) in the definition of a walk is satisfied because no edge in $H_{e}^{z}$ contains both $z$ and $z^{\prime}$.

We shall call the walk $P$ in $H$, obtained from a walk $P^{\prime}$ in $H_{e}^{z}$ in the way described in Lemma 12, the walk derived from $P^{\prime}$. Let us make a few observations on the derived walks. First, if a walk $P^{\prime}$ is a tour (resp. Euler tour) in $H_{e}^{z}$, then the derived walk is a tour (resp. Euler tour) in $H$. Second, if $P^{\prime}$ is a $z z^{\prime}$-walk (resp. Euler $z z^{\prime}$-walk) in $H_{e}^{z}$, then the derived walk is a tour (resp. Euler tour) in $H$. Third, if a walk $P^{\prime}$ passes through at least one of the vertices $z$ or $z^{\prime}$ in $H_{e}^{z}$, then the derived walk passes through the vertex $z$ in $H$.

In the next three lemmas we study the relationship between the skeletons of hypergraphs $H$ and $H_{e}^{z}$. Let us recall that by $S(H)$ we denote the skeleton of $H$.

\section{Lemma 13}

$$
V(S(H))-\{z\} \subseteq V\left(S\left(H_{e}^{z}\right)\right) .
$$

Proof. Let $e_{1}, e_{2}, \ldots, e_{m}$ be an $(e, z)$-ordering of the set of edges of $H$ such that $e=$ $x y z=e_{i}$ and let $u \in V(S(H))-\{z\}$. Then there are edges $e_{\ell}$ and $e_{j}$ in $H$ such that $e_{\ell} \cap e_{j}=u v, \ell<j$, for some vertex $v$ in $H$. 


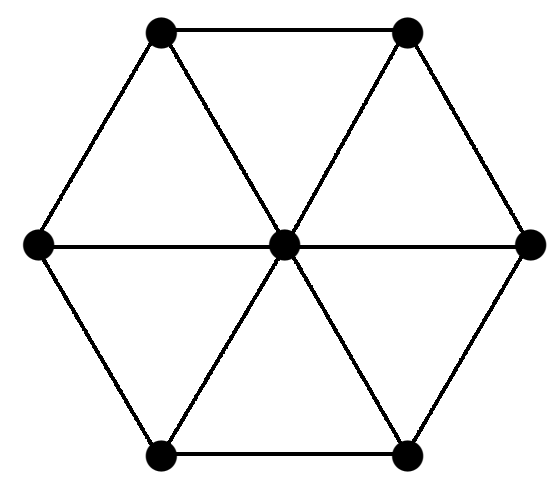

Figure 7: The wheel $W_{6}$ (the triangles in this figure are the edges of the wheel).

Suppose first that $v \neq z$. Then $e_{\ell}^{\prime} \cap e_{j}^{\prime} \supseteq\left(e_{\ell}-\{z\}\right) \cap\left(e_{j}-\{z\}\right)=\left(e_{\ell} \cap e_{j}\right)-\{z\}=$ $e_{\ell} \cap e_{j}=u v$, so $u v$ is an edge in $S\left(H_{e}^{z}\right)$ and, consequently, $u \in V\left(S\left(H_{e}^{z}\right)\right)$.

It remains to consider the case when $v=z$. If $\ell<i$, then, by Lemma $10, j \leqslant i$. For $\ell<i$ and $j<i, e_{\ell}^{\prime} \cap e_{j}^{\prime}=e_{\ell} \cap e_{j}=u v$, so we are done. If $\ell<i$ and $j=i$, then $u=x$ or $u=y$. In both cases $u \in V\left(S\left(H_{e}^{z}\right)\right)$ because $x y=e-\{z\}$ is an edge in $S\left(H_{e}^{z}\right)$, by the condition (i) in the definition of an $(e, z)$-ordering. Finally, let $i \leqslant \ell<j$. Then $e_{\ell}^{\prime} \cap e_{j}^{\prime}=\left(\left(e_{\ell}-\{z\}\right) \cup\left\{z^{\prime}\right\}\right) \cap\left(\left(e_{j}-\{z\}\right) \cup\left\{z^{\prime}\right\}\right)=\left(\left(e_{\ell} \cap e_{j}\right)-\{z\}\right) \cup\left\{z^{\prime}\right\}=u z^{\prime}$, so $u z^{\prime}$ is an edge in $S\left(H_{e}^{z}\right)$ and, consequently, $u \in V\left(S\left(H_{e}^{z}\right)\right)$.

Lemma 14 If $H_{e}^{z}$ is the hypergraph defined for some nonterminal $(e, z)$-ordering of the set of edges of a strongly connected 3-uniform hypergraph $H$, then

$$
(V(S(H))-\{z\}) \cup\left\{z^{\prime}\right\} \subseteq V\left(S\left(H_{e}^{z}\right)\right) .
$$

Proof. Let $e_{1}, e_{2}, \ldots, e_{m}$ be a nonterminal $(e, z)$-ordering of the set of edges of $H$ and let $e=x y z=e_{i}$, where $i<m$. By Lemma 13, it suffices to show that $z^{\prime} \in V\left(S\left(H_{e}^{z}\right)\right)$. We observe that the edge $e_{i+1}$ contains $x z$ or $y z$. Otherwise, by the definition of a tree-like ordering of the set of edges and the condition (i) in the definition of an $(e, z)$-ordering, $e_{i+1}$ has 2 vertices in common with some edge $e_{j}, j<i$, which contradicts Lemma 10 . Thus, $x z$ or $y z$ is a subset of both $e_{i}$ and $e_{i+1}$. Consequently, $x z^{\prime}$ or $y z^{\prime}$ is a subset of both $e_{i}^{\prime}$ and $e_{i+1}^{\prime}$, which proves that $z^{\prime} \in V\left(S\left(H_{e}^{z}\right)\right)$.

In the next lemma a hypergraph that we call a wheel occurs. The definition of a wheel has been given in the Introduction. The wheel $W_{6}$ is depicted in Figure 7.

Lemma 15 If a strongly connected 3-uniform hypergraph $H$ has a substantial edge and all $(e, z)$-orderings of edges of $H$ are terminal, then there exists an $(e, z)$-ordering of the set of edges of $H$ such that for the hypergraph $H_{e}^{z}$ defined for this ordering

$$
V(S(H)) \subseteq V\left(S\left(H_{e}^{z}\right)\right)
$$

unless $H$ is a wheel. 
Proof. Let us observe that if $H$ has less than 3 edges, then no edge is substantial. Therefore, we shall assume that $m=|E(H)|>2$.

If there is an $(e, z)$-ordering of the set of edges of $H$ such that $z \notin V(S(H))$, then, by Lemma 13, $V(S(H)) \subseteq V\left(S\left(H_{e}^{z}\right)\right)$, for the hypergraph $H_{e}^{z}$ defined for this $(e, z)$-ordering.

Therefore, we shall assume in the rest of the proof that $z \in V(S(H))$, for every $(e, z)$ ordering of the set of edges of $H$. By Lemma 13, it suffices to show that $z \in V\left(S\left(H_{e}^{z}\right)\right)$, for some $(e, z)$-ordering of the set of edges of $H$, if $H$ is not a wheel.

We consider an arbitrary $(e, z)$-ordering $e_{1}, e_{2}, \ldots, e_{m}$ of the set of edges of $H$. By our assumptions it is terminal. Let $e=e_{m}=x y z$. By Lemma 1, the hypergraph $H^{\prime}=H-e$ is strongly connected. We can assume that $z \notin V\left(S\left(H^{\prime}\right)\right)$ because otherwise $z \in V\left(S\left(H^{\prime}\right)\right) \subseteq V\left(S\left(H_{e}^{z}\right)\right)$, so we are done. As $z \in V(S(H))$, xz or $y z$ are edges of $S(H)$. Assume first that both $x z$ and $y z$ are edges of $S(H)$. Since $z \notin V\left(S\left(H^{\prime}\right)\right)$, there is a unique edge $x z u$ (resp. $y z v$ ) in $H^{\prime}$ that contains the pair $x z$ (resp. $y z$ ). By the strong connectivity of $H^{\prime}$ and the fact that $\left|E\left(H^{\prime}\right)\right|>1, x u$ is an edge in $S\left(H^{\prime}\right)$ (as $z \notin V\left(S\left(H^{\prime}\right)\right)$ and neither $z x$ nor $z u$ is an edge in $\left.S\left(H^{\prime}\right)\right)$. We are done in this case by Lemma 13 because $e_{1}, e_{2}, \ldots, e_{m}$ is also an $(e, x)$-ordering of the set of edges of $H$ and $x \in V\left(S\left(H^{\prime}\right)\right) \subseteq V\left(S\left(H_{e}^{x}\right)\right)$.

Thus, we can assume that exactly one of the pairs $x z$ or $y z$, say $x z$, is an edge in $S(H)$. Consequently, as $z \notin V\left(S\left(H^{\prime}\right)\right), d_{S(H)}(z)=1$ and there is a unique edge in $H^{\prime}$ that contains the pair $x z$.

We observe now that, as $x z$ is contained in an edge of $H^{\prime}, e_{1}, e_{2}, \ldots, e_{m}$ is also an $(e, y)$ ordering of the set of edges of $H$. Using the same argument for this $(e, y)$-ordering as for the $(e, z)$-ordering considered above, we show that we can assume that $y \notin V\left(S\left(H^{\prime}\right)\right)$ and $d_{S(H)}(y)=1$ because otherwise we are done. For the same reason we can assume that exactly one of the pairs $y x$ or $y z$ is an edge in $S(H)$. However, $y z$ can not be an edge in $S(H)$ because $d_{S(H)}(z)=1$ and $x z$ is an edge in $S(H)$. Hence $y x$ is the only edge in $S(H)$ that contains $y$. Moreover, since $y \notin V\left(S\left(H^{\prime}\right)\right)$, there is a unique edge in $H^{\prime}$ that contains the pair $y x$.

Summarizing, it remains to prove our Lemma for 3-uniform strongly connected hypergraphs $H$ satisfying the assumptions of this Lemma such that for every substantial edge $e$ in $H$

(i) e contains exactly two edges of $S(H)$,

(ii) exactly one of the vertices of $e$ has the degree larger than 1 in $S(H)$, and

(iii) e has exactly two neighbors in the strong connectivity graph $G(H)$.

As the hypergraph $H^{\prime}$ is strongly connected, the strong connectivity graph $G\left(H^{\prime}\right)$ obtained from $G(H)$ by removing the vertex $e$ is connected. Therefore, by (iii), the vertex $e$ belongs to some cycle $C$ in $G(H)$. Moreover, both neighbors of $e$, say $f$ and $h$, in $G(H)$ belong to the cycle $C$ too. Clearly, by (i) and (iii), $f \cap e \neq h \cap e$. As $f$ is on a cycle in $G(H)$, the hypergraph $H^{\prime \prime}=H-f$ is strongly connected. Let $f_{1}, f_{2}, \ldots, f_{m-1}$ be a tree-like ordering of the set of edges of $H^{\prime \prime}$. Let $g$ be a neighbor of $f$ in $G(H)$ different from $e$ (it exists because $f$ belongs to a cycle in $G(H)$ ). We observe that $f \subseteq e \cup g$. Indeed, 
otherwise $f \cap e=f \cap g=g \cap e$, so $g$ is a neighbor of $e$ in $G(H)$ and, consequently, $g=h$. We get a contradiction with the inequality $f \cap e \neq h \cap e$. The inclusion $f \subseteq e \cup g$ implies that $f$ is contained in the vertex set of $H^{\prime \prime}$. Consequently, $f_{1}, f_{2}, \ldots, f_{m-1}, f_{m}=f$ is an $(f, v)$-ordering of the set of edges for some vertex $v \in f$. Thus the edge $f$ is substantial.

We have shown that if the Lemma does not hold for a 3-uniform strongly connected hypergraph $H$, then every substantial edge in $H$ has exactly two neighbors in the graph $G(H)$ which are substantial edges too. Since, by an assumption of this Lemma, there is a substantial edge in $H$, this fact implies that some set of substantial edges forms a component in the strong connectivity graph $G(H)$ which is a cycle. Consequently, as $H$ is strongly connected, the graph $G(H)$ itself must be a cycle whose all vertices are substantial edges in $H$. To complete the proof we show that $H$ is a wheel. To this end we prove that all edges of $H$ contain a common vertex. We shall use induction. Let $e_{1}, e_{2}, \ldots, e_{m}$ be the edges of $H$ in the order they occur in the cycle $G(H)$. We denote by $x$ the vertex of $e_{1}$ which belongs to more than one edge in $S(H)$. Its existence is guaranteed by the condition (ii). Suppose that $x$ belongs to each of the edges $e_{1}, e_{2}, \ldots, e_{i}, i<m$. We shall prove that $x \in e_{i+1}$. Let $e_{i}=x u w$. By conditions (i), (ii), and the definition of $x, x u$ and $x w$ are edges in $S(H)$ and the pair $u w$ is not an edge in $S(H)$. Since $e_{i+1}$ is a neighbor of $e_{i}$ in $G(H), e_{i+1}$ and $e_{i}$ have two vertices in common. If $x \notin e_{i+1}$, then $e_{i+1} \cap e_{i}=u w$. Then, however, $u w$ is an edge in $S(H)$, a contradiction. Thus, $x \in e_{i+1}$. By induction, $x$ belongs to every edge in $H$. Consequently, $H$ is a wheel which completes our proof.

Lemma 16 Let $H \in \mathcal{H}$. The vertex set of the skeleton of $H$ is equal to the set of vertices of degree greater than 1 in $H$.

Proof. We apply induction on the size of $H$. If $H$ has one edge only, then we are trivially done.

Let us assume now that $|E(H)|>1$. The hypergraph $H$ is of the form $H^{\prime}+e$, where $H^{\prime} \in \mathcal{H}$ and $e \in E(H)$. Moreover, there is an edge $f \in E\left(H^{\prime}\right)$ such that $f$ and $e$ have two vertices, say $u$ and $v$, in common. By the definition of the class $\mathcal{H}$, the set of vertices of the skeleton of $H$ is a union of the set vertices of the skeleton of $H^{\prime}$ and $\{u, v\}$. Similarly, the set of vertices of degree greater than 1 in $H$ is the union of the set of vertices of degree greater than 1 in $H^{\prime}$ and $\{u, v\}$. We are now done by the induction hypothesis.

Due to Lemma 16, we can now extend the definition of a good walk, which has so far been defined for 3-uniform hypergraphs in $\mathcal{H}$ only, to arbitrary 3-uniform hypergraphs. We shall call an Euler walk in a 3-uniform hypergraph $H$ good if it passes through every vertex of the skeleton of $H$. By Lemma 16, this definition is consistent with the definition of a good walk for hypergraphs in $\mathcal{H}$.

The following lemma (perhaps interesting by itself) gives a simple characterization of strongly connected 3-unifrom hypergraphs that have a good tour.

Lemma 17 A strongly connected 3-uniform hypergraph $H$ has a good tour if and only if $H$ is neither a member of $\mathcal{H}$ having all vertices of odd degree nor a wheel. 
Proof. Suppose a wheel $W_{\ell}, \ell \geqslant 3$, has a good tour. Since the skeleton of the wheel $W_{\ell}$ has $\ell+1$ vertices, a good tour in $W_{\ell}$ must contain at least $\ell+1$ edges. We get a contradiction because the wheel $W_{\ell}$ has only $\ell$ edges. Thus wheels do not have good tours. By Corollary 1, hypergraphs in $\mathcal{H}$ with all vertices of odd degree do not have good tours either. This completes the proof of necessity.

To prove sufficiency, we apply induction on the number $s=|E(H)|-|V(H)|+2$. By Lemma 2, for every strongly connected 3-uniform hypergraph $H, s=|E(H)|-|V(H)|+$ $2 \geqslant 0$. For $s=0$ our Lemma follows from Lemma 3 and Corollary 1.

Let $H$ be a strongly connected 3-uniform hypergraph such that $s=|E(H)|-|V(H)|+$ $2>0$ which is neither a member of $\mathcal{H}$ having all vertices of odd degree nor a wheel. By Lemma 9 , there is an $(e, z)$-ordering of the set of edges of $H$, for some $e \in E(H)$ and $z \in e$, so $H$ contains a substantial edge.

Let us consider the hypergraph $H_{e}^{z}$ defined for some $(e, z)$-ordering $e_{1}, e_{2}, \ldots, e_{m}$ of the set of edges of $H$ which is additionally nonterminal, if a nonterminal $(e, z)$-ordering exits. The hypergraph $H_{e}^{z}$ is strongly connected, by Lemma 11. Moreover, $\left|E\left(H_{e}^{z}\right)\right|-\left|V\left(H_{e}^{z}\right)\right|+$ $2=|E(H)|-(|V(H)|+1)+2<s$. By the induction hypothesis, $H_{e}^{z}$ has a good tour $C^{\prime}$ unless it is a member of $\mathcal{H}$ having all vertices of odd degree or a wheel. Suppose first $H_{e}^{z}$ is none of these two kinds of hypergraphs. Let $C$ be the tour in $H$ derived from $C^{\prime}$. If $H_{e}^{z}$ is defined for a nonterminal $(e, z)$-ordering of the set of edges of $H$, then, by Lemmas 12 and 14, $C$ is a tour in $H$ passing through every vertex of $S(H)$, so it is a good tour in $H$. If all $(e, z)$-orderings of the set of edges in $H$ are terminal, then, by Lemma 15, there exists an $(e, z)$-ordering of the set of edges of $H$ such that, for the hypergraph $H_{e}^{z}$ defined for this ordering, $V(S(H)) \subseteq V\left(S\left(H_{e}^{z}\right)\right)$ because $H$ is not a wheel. In this case too the tour $C$ is good in $H$, by Lemma 12 .

It remains to consider the case when $H_{e}^{z}$ is a wheel or a member of $\mathcal{H}$ having no vertex of even degree.

In the former case let $C^{\prime}$ be an Euler tour in the wheel $H_{e}^{z}$ which passes through every vertex of $H_{e}^{z}$ except $z^{\prime}$. (One can readily verify that for any vertex $v$ of a wheel there exists an Euler tour passing through all vertices of the wheel except $v$.) It follows from the condition (ii) in the definition of an $(e, z)$-ordering and the definition of the hypergraph $H_{e}^{z}$ that $z$ is a vertex of $H_{e}^{z}$, so $z$ is a vertex of $C^{\prime}$ too. By Lemma 12, the tour $C$ derived from $C^{\prime}$ passes through all vertices of $H$, so it is a good tour in $H$.

In the latter case, by Corollary 2 and Lemma 5, there is a good $z z^{\prime}$-walk $P^{\prime}$ in $H_{e}^{z}$. It follows from Lemmas 12 and 13 that the walk $P$ derived from $P^{\prime}$ is a good tour in $H$.

Proof of Theorem 6. We shall prove a stronger result that $H$ has a good tour.

We apply induction on the number of strong components of $H$. If $H$ has one strong component only, then we are done by Lemma 17.

Let us assume now that $H$ has at least two strong components. Let $G_{s}(H)$ be the graph whose vertex set is the set of strong components of $H$ and two vertices $K$ and $L$ of $G_{s}(H)$ are joined by an edge in $G_{s}(H)$ if and only if the skeletons of the strong components $K$ and $L$ have a common vertex. The skeleton $S(H)$ of $H$ is the union of the skeletons of its components, so the graph $G_{s}(H)$ is connected because, by our assumption, the skeleton of $H$ is connected. Let $K$ be a leaf of some spanning tree in $G_{s}(H)$. The graph 


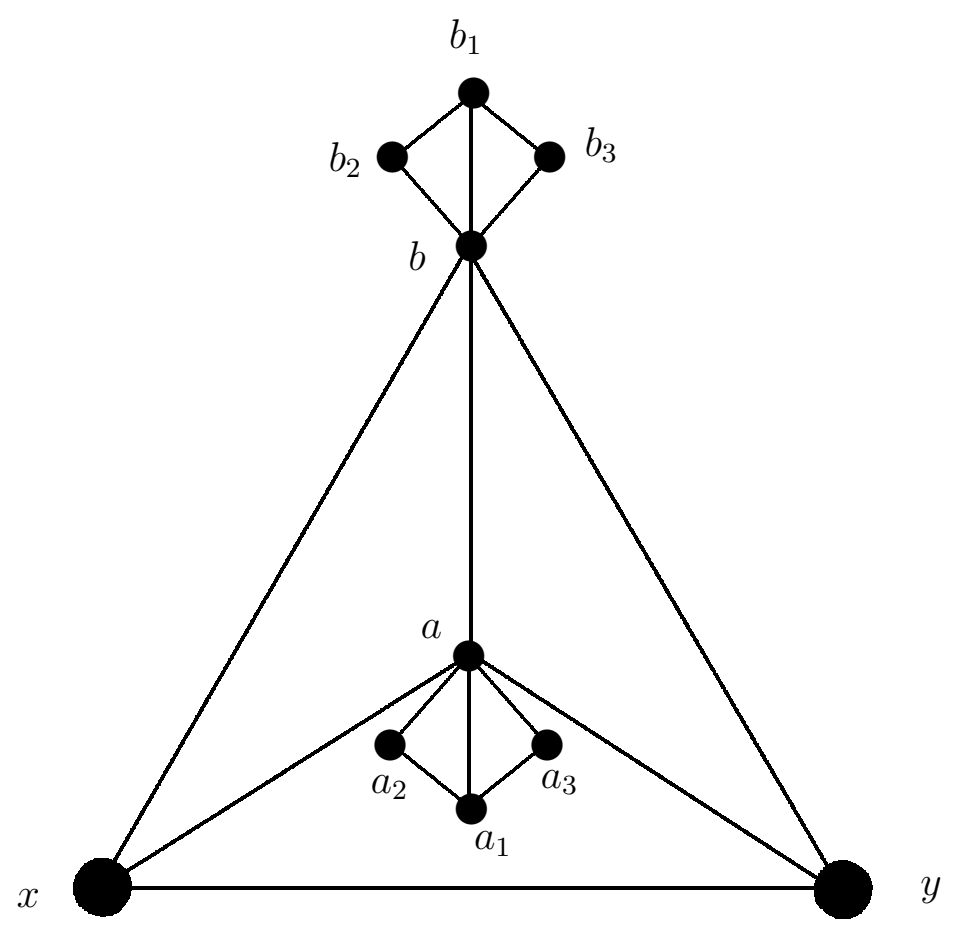

Figure 8:

$G_{s}(H)-K$, obtained from $G_{s}(H)$ by removing the vertex $K$, is connected. Therefore, the hypergraph $F=H-E(K)$ has a connected skeleton because, by Lemma 8, the skeleton of each strongly connected component of $H$ is connected. By the induction hypothesis, $F$ has a good tour, say $C$, and $K$ has a good tour, say $D$. Let $v$ be a common vertex of the skeletons of $F$ and $K$. Then both tours $C$ and $D$ pass through $v$, so $v C v D v$ is a good tour in $H$.

In the proof of NP-completeness of the problem of deciding if a 3-uniform hypergraph with a connected skeleton has an Euler tour (Theorem 7) we shall use some special hypergraphs that we introduce now.

Let $F$ be a 3-uniform hypergraph with the vertex set $V(F)=\left\{x, y, a, b, a_{1}, a_{2}, a_{3}, b_{1}, b_{2}\right.$, $\left.b_{3}\right\}$ and the edge set $E(F)=\left\{x y a, x a b, y a b, a a_{1} a_{2}, a a_{1} a_{3}, b b_{1} b_{2}, b b_{1} b_{3}\right\}$ (see Figure 8). We call the vertices $x$ and $y$ in $F$ the connectors of $F$.

One can readily verify that the hypergraph $F$ has the following property.

Proposition 6 Let the hypergraph $F$ be a subhypergraph of a 3-uniform hypergraph $H$ such that no vertices of $F$, except possibly the connectors, belong to any edge of $H$ which is not an edge in F. In every Euler tour in $H$ the edges of $F$ occur as consecutive edges.

Let us denote by $F^{*}$ the hypergraph obtained from three vertex-disjoint copies of the hypergraphs $F$ by identifying their connectors $y$ and adding three new vertices $y_{1}, y_{2}, y_{3}$ 


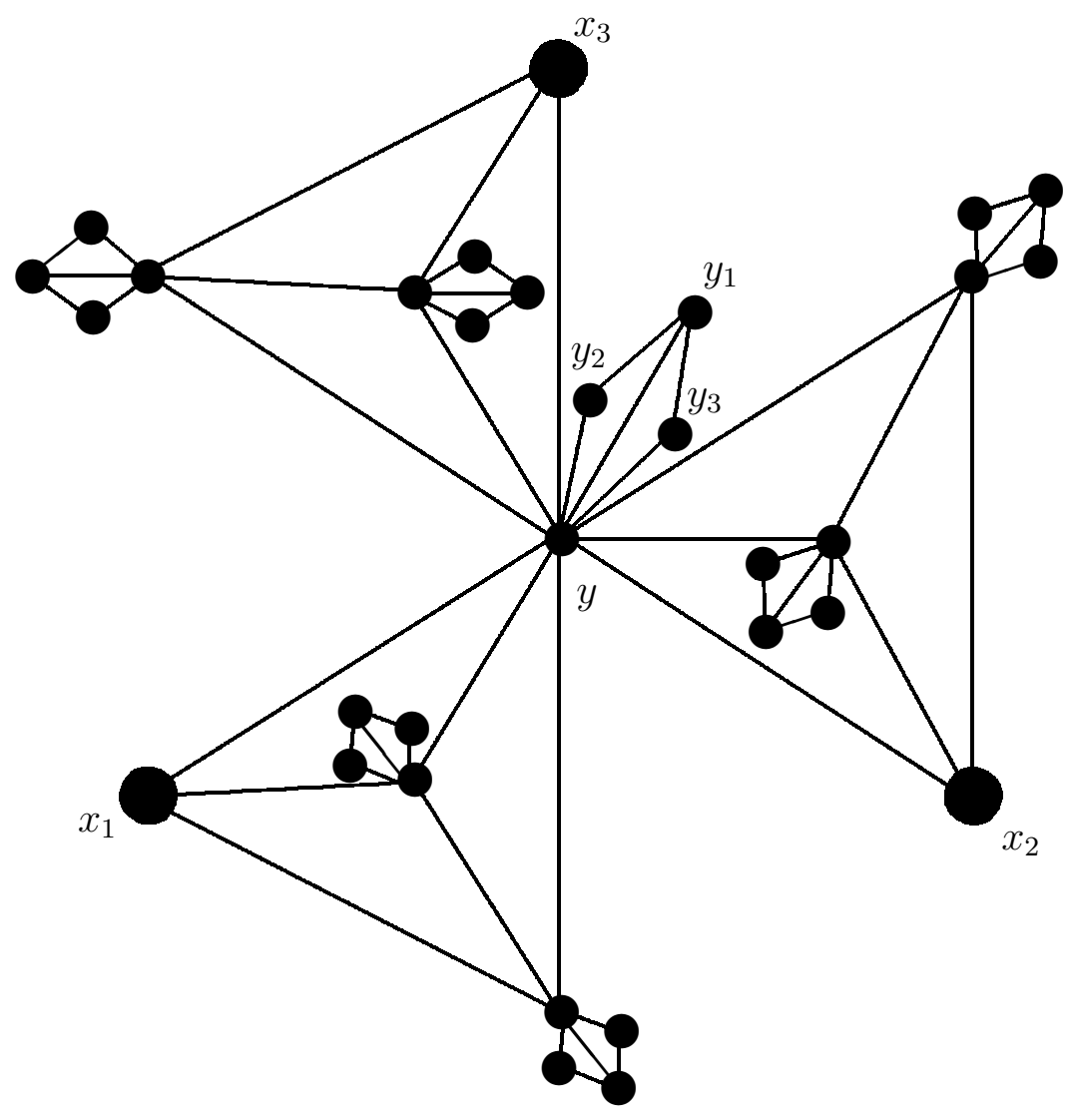

Figure 9:

and two edges $y y_{1} y_{2}, y y_{1} y_{3}$. Let $x_{1}, x_{2}$, and $x_{3}$ be the other connectors of the three copies of $F$ (see Figure 9). We call these three vertices $x_{1}, x_{2}, x_{3}$ the connectors of $F^{*}$. We shall need the following easy to prove property of the hypergraph $F^{*}$.

Proposition 7 For every two different connectors $v$ and $w$ in $F^{*}$, there is an Euler vw-walk in $F^{*}$.

We call a 3-uniform hypergraph $H$ connected if, for every pair of vertices $u, v \in V(H)$, there is a $u v$-walk in $H$. Obviously, by Theorem 1, the problem of existence of an Euler tour in a connected 3-uniform hypergraph is NP-complete.

Proof of Theorem 7. Clearly, the problem of determining if a 3-uniform hypergraph with a connected skeleton has an Euler tour belongs to the class NP.

We shall show a reduction from the problem of existence of an Euler tour in a connected 3-uniform hypergraph to our problem.

Let $H$ be a connected 3-uniform hypergraph. We replace each edge $e=x_{1} x_{2} x_{3}$ of $H$ by a copy $F_{e}^{*}$ of the hypergraph $F^{*}$ with the connectors $x_{1}, x_{2}$, and $x_{3}$. Let $y_{e}$ be the vertex $y$ in $F_{e}^{*}$. We denote the 3 -uniform hypergraph obtained this way by $H^{*}$. We observe that the hypergraph $H^{*}$ has a connected skeleton. Clearly, the construction of $H^{*}$ can be done in polynomial time with respect to the size of $H$. 
To complete the proof, it suffices to show that $H$ has an Euler tour if and only if $H^{*}$ has one.

Let $C$ be an Euler tour in $H$. Moreover, let $e$ be any edge in $H$ and let $v$ and $w$ be vertices in $H$ using which the tour $C$ traverses $e$. We denote by $P_{e}^{v w}$ an Euler $v w$-walk in $F_{e}^{*}$ whose existence is guaranteed by Proposition 7 . We replace in $C$ every subsequence vew by $v P_{e}^{v w} w$. Clearly, the sequence obtained this way is an Euler tour in $H^{*}$.

Conversely, assume the hypergraph $H^{*}$ has an Euler tour $C^{*}$. It follows from the definition of the hypergraph $H^{*}$ that $C^{*}$ passes through every vertex $y_{e}$, where $e$ is an edge in $H$. Consequently, by Proposition 6, for every edge $e$ in $H$ there is exactly one subsequence of $C^{*}$ of the form $v Q_{e} y_{e} R_{e} w$ such that $v$ and $w$ are connectors of $F_{e}^{*}$, the sequence $Q_{e}$ contains all the edges of the copy of $F$ with connectors $v$ and $y_{e}$ being a part of $F_{e}^{*}$, and $R_{e}$ contains all the edges of the copy of $F$ with connectors $y_{e}$ and $w$ being a part of $F_{e}^{*}\left(Q_{e}\right.$ or $R_{e}$ may or may not also contain all edges of the third copy of $F$ in $\left.F_{e}^{*}\right)$. We replace each such subsequence $v Q_{e} y_{e} R_{e} w$ of $C^{*}$ by the sequence $v e w$. In the resulting sequence, say $C^{\prime}$, every edge of the hypergraph $H$ occurs exactly once. The sequence $C^{\prime}$ may still contain some subsequences of the form $u P_{e} u$, where $e$ is an edge in $H$ and $P_{e}$ contains all the edges of some copy of $F$ with connectors $u$ and $y_{e}$ being a part of $F_{e}^{*}$. We replace such subsequences by the sequence $u$. The resulting sequence $C$ is an Euler tour in $H$.

\section{Acknowledgements}

We thank dr Armin Fügenschuh for introducing us to the subject of this paper and for pointing out some relevant references.

This research was supported by the Polish Ministry of Science and Higher Education in 2009-2010 under grant No. 3674/B/H03/2009/36.

\section{References}

[1] E. M. Arkin, M. Held, J. S. B. Mitchell and S. S. Skiena, Hamilton triangulations for fast rendering, Algorithms - ESA '94 (Utrecht), Lecture Notes in Comput. Sci. 855 (1994) 36-47.

[2] J. J. Bartholdi III and P. Goldsman, Continuous indexing of hierarchical subdivisions of the globe, Int. J. Geographical Information Science 15 (6) (2001) 489-522.

[3] J. J. Bartholdi III and P. Goldsman, Multiresolution indexing of triangulated irregular networks, IEEE Transactions on Visualization and Computer Graphics 10 (3) (2004) 484-495.

[4] J. J. Bartholdi III and P. Goldsman, The vertex-adjacency dual of a triangulated irregular network has a Hamiltonian tour, Operations Research Letters 32 (2004) 304-308.

[5] J. J. Bartholdi III and P. Goldsman, Vertex-labeling algorithms for the Hilbert spacefilling curve, Softw. Pract. Exper. 31 (2001) 395-408. 
[6] C. Berge, Hypergraphs, Combinatorics of Finite Sets, North-Holland, 1989.

[7] M. R. Garey and D. S. Johnson, Computers and Intractability: A Guide to the Theory of NP-Completeness, W. H. Freeman, 1979.

[8] A. Gyárfás, J. Lehel, G. N. Sárközy and R. H. Schelp, Monochromatic Hamiltonian Berge-cycles in colored complete uniform hypergraphs, J. Combinatorial Theory Ser. B 98 (2008) 342-358.

[9] G. Y. Katona and H. A. Kierstead, Hamiltonian chains in hypergraphs, J. Graph Theory 30 (3) (1999) 205-212.

[10] P. Keevash, D. Kühn, R. Mycroft and D. Osthus, Loose Hamilton cycles in hypergraphs, 1-21, preprint.

[11] D. Kühn and D. Osthus, Loose Hamilton cycles in 3-uniform hypergraphs of large minimum degree, J. Combinatorial Theory Ser. B 96 (2006) 767-821.

[12] V. Rödl, A. Ruciński and E. Szemerédi, A Dirac-type theorem for 3-uniform hypergraphs, Combinatorics, Probability and Computing 15 (1-2) (2006) 229-251.

[13] V. Rödl, A. Ruciński and E. Szemerédi, An approximate Dirac theorem for $k$-uniform hypergraphs, Combinatorica 28 (2) (2008) 229-260.

[14] D. L. Wilson, Polyhedral Methods for Piecewise-Linear Functions, Ph.D. Thesis, University of Kentucky, Lexington, 1998. 